\title{
On several new or poorly-known Oriental Paradoxosomatidae (Diplopoda: Polydesmida), XIV
}

\section{О несколыких новых или плохоизученных ориенталыных Paradoxosomatidae (Diplopoda: Polydesmida), XIV}

\author{
S.I. Golovatch \\ С.И. Головач
}

Institute for Problems of Ecology and Evolution, Russian Academy of Sciences, Leninsky prospekt 33, Moscow 119071 Russia. E-mail: sgolovatch@yandex.ru

Институт проблем экологии и эволюции РАН, Ленинский проспект, 33, Москва 119071 Россия.

KEY WORDS: Diplopoda, Polydesmida, Paradoxosomatidae, taxonomy, new records, new species, China.

КЛЮЧЕВЫЕ СЛОВА: Diplopoda, Polydesmida, Paradoxosomatidae, таксономия, новые находки, новые виды, Китай.

ABSTRACT. This contribution is devoted to new records of a few known, and to descriptions of the following nine new, species from China: Gonobelus pentaspinus sp.n., Kronopolites semirugosus sp.n., Hedinomorpha nigra sp.n., Sellanucheza typica sp.n., Sigipinius complex sp.n. and $S$. simplex sp.n., all from Sichuan, $S$. kabaki sp.n. from Xinjiang, as well as $S$. campanuliformis sp.n. and Hedinomorpha subnigra sp.n., both latter species from Yunnan. The diagnosis and systematic position of Sigipinius Hoffman, 1961 are refined, the type species, S. grahami Hoffman, 1961 , is redescribed, based on abundant near-topotype material, whereas this genus is transferred to the tribe Sundaninini, comb.n., and considered as a new subjective senior synonym of Chinomorpha Golovatch, 2011, syn.n. Keys to all currently known eight, six and six species of Hedinomorpha Verhoeff, 1933, Sellanuche$z a$ Enghoff, Golovatch et Nguyen Duc, 2004 and Sigipinius are given, respectively. Additional records of Inversispina erectispina Golovatch, 2012 are presented from Sichuan, with remarks on variability.

РЕЗЮМЕ. Данное сообщение посвящено находкам нескольких известных, а также описаниям следующих девяти новых видов из Китая: Gonobelus pentaspinus sp.n., Kronopolites semirugosus sp.n., Hedinomorpha nigra sp.n., Sellanucheza typica sp.n., Sigipinius complex sp.n. и $S$. simplex sp.n., все из Сычуани, $S$. kabaki sp.n. из Синьзяня, а также $S$. campanuliformis sp.n. и Hedinomorpha subnigra sp.n., оба последних вида из Юннани. Уточнены диагноз и систематическое положение рода Sigipinius Hoffman, 1961, по почти топотипам переописан типовой вид $S$. graha$m i$ Hoffman, 1961, а сам род перенесен в состав трибы Sundaninini, comb.n., и считается новым субъективным старшим синонимом рода Chinomorpha Golovatch, 2011, syn.n. Даны ключи соответственно для всех восьми, шести и шести ныне известных видов родов Hedinomorpha Verhoeff, 1933, Sellanucheza Enghoff, Golovatch et Nguyen Duc, 2004 and Sigipinius. Представлены новые находки вида Inversispina erectispina Golovatch, 2012 в Сычуани с замечаниями о его изменчивости.

\section{Introduction}

This paper is devoted to new records of a few known, as well as to descriptions of nine new, species of paradoxosomatid millipedes from China. The material, chiefly collected by several Russian entomologists, has largely been deposited in the collection of the Zoological Museum, Moscow State University, Russia (ZMUM), with only a few samples housed in the Zoological Institute, Russian Academy of Sciences, St. Petersburg (ZISP), Russia, and the National Museum of Natural History, Sofia (NMNHS), Bulgaria, as indicated below.

\section{Taxonomic part}

Oxidus gracilis (C.L. Koch, 1847)

MATERIAL. $10^{7}$ (ZMUM), China, Sichuan Prov., Maoxian

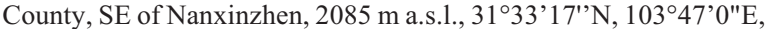
cultivated land, houses and gardens, 28.06.2012, leg. I.A. Belousov \& G.E. Davidian. $1 \sigma^{7}, 1$ ( (ZMUM), Xinjiang Uygur Prov., Kunges River, $\mathrm{N}$ of Kunges, $840 \mathrm{~m}$ a.s.1., $43^{\circ} 31^{\prime} 12^{\prime \prime N}, 83^{\circ} 15^{\prime} 22^{\prime \prime E}$, 15.07.2012, leg. I.I. Kabak.

REMARKS. This subcosmopolitan, definitely anthropochore species is very common all over China [e.g. Golovatch, 2012, 2013]. The record above 2000 $\mathrm{m}$, i.e. quite high in the mountains, is remarkable and must lie close to the uppermost range limit of $O$. gracilis. 

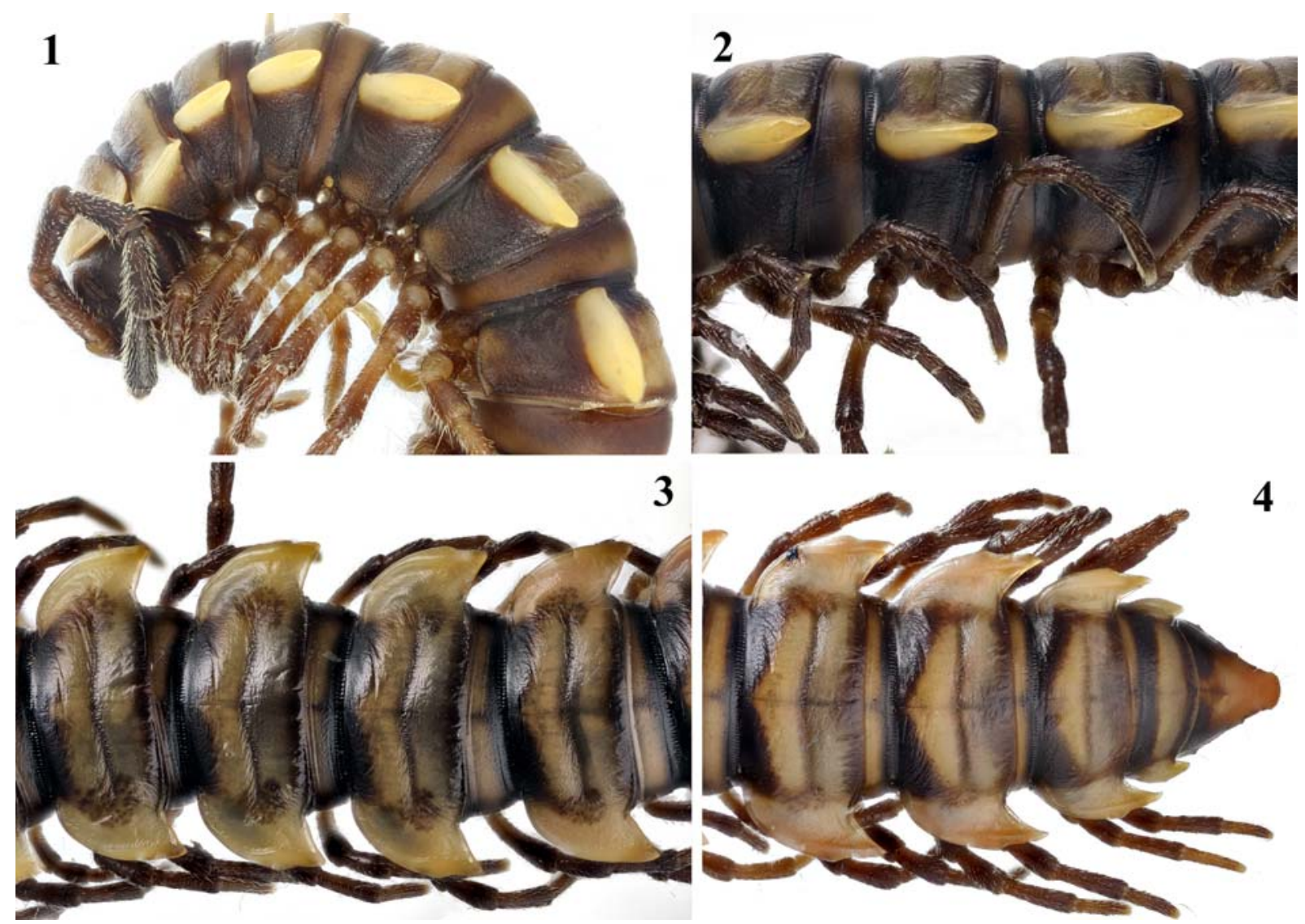

Figs 1-4. Antheromorpha rosea Golovatch, 2013, $\sigma^{\text {T}}: 1$ - anterior body part, lateral view; $2 \& 3$ - midbody segments, lateral and dorsal views, respectively; 4 - caudal body part, dorsal view. Photos taken by K. Makarov, not to scale.

Рис. 1-4. Antheromorpha rosea Golovatch, 2013, О7: 1 - передняя часть тела, сбоку; 2 и 3 - среднетуловищные сегменты, соответственно сбоку и сверху; 4 - задняя часть тела, сверху. Фото сделаны К. Макаровым, без масштаба.

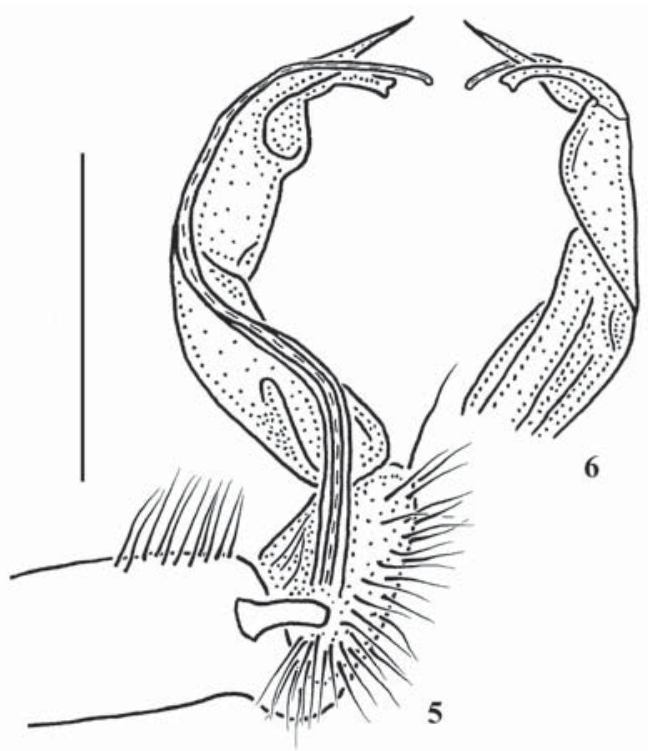

Figs 5 \& 6. Antheromorpha rosea Golovatch, 2013, $0^{7}$ : left gonopod, mesal and lateral views, respectively. Scale bar: $1.0 \mathrm{~mm}$.

Рис. 5 и 6. Antheromorpha rosea Golovatch, 2013, о?: левый гонопод, соответственно изнутри и сбоку. Масштаб 1,0 мм.
Antheromorpha rosea Golovatch, 2013

Figs 1-6.

MATERIAL. $1 \sigma^{7}, 1$ (NMNHS), China, Yunnan Prov., Bao Shan Distr., near Hemu, Gaoligong Mount Biosphere Nature Reserve, near Cave Bian Fu Dong No. 2 (= Bat Cave), $24.98442^{\circ} \mathrm{N}$, $98.80489^{\circ} \mathrm{E}, 1200 \mathrm{~m}$ a.s.1., 29.10 .2011 , leg. B. Petrov.

REMARKS. This near-topotype sample agrees fully with the original description [Golovatch, 2013]. I illustrate it again to document the present record (Figs $1-6)$.

Inversispina erectispina Golovatch, 2012 Figs 7-19.

MATERIAL. $3 \sigma^{7} \sigma^{7}, 2$, 2 (ZMUM), China, Sichuan Prov., NW of Mianning, $2955 \mathrm{~m}$ a.s.1., $28^{\circ} 39^{\prime} 13^{\prime \prime} \mathrm{N}, 101^{\circ} 58^{\prime} 34^{\prime \prime} \mathrm{E}$, broadleaved forest, 9.06.2012; $1 \sigma^{7}, 1+$ (NMNHS), same locality, $3570 \mathrm{~m}$ a.s.1., $28^{\circ} 40^{\prime} 25^{\prime \prime} \mathrm{N}, 102^{\circ} 02^{\prime} 01^{\prime \prime} \mathrm{E}$, broadleaved forest, 6.06. 2012, $2 \mathrm{O}^{7} \mathrm{O}^{7}$ (ZMUM), same locality, $3895 \mathrm{~m}$ a.s.1., $28^{\circ} 39^{\prime} 51^{\prime \prime} \mathrm{N}, 102^{\circ} 00^{\prime} 22^{\prime \prime} \mathrm{E}$, broadleaved forest, 8.06.2012; $1 \mathrm{O}^{2}, 1 \%$ (ZISP), same locality, 4035 $\mathrm{m}$ a.s.1., broadleaved forest, $28^{\circ} 40^{\prime} 03^{\prime \prime} \mathrm{N}, 102^{\circ} 00^{\prime} 56^{\prime \prime} \mathrm{E}, 7.06 .2012 ; 3$ $\mathrm{O}^{7} \mathrm{O}^{7}, 1$ ( (ZMUM), same locality, $2955 \mathrm{~m}$ a.s.l., broadleaved forest, $28^{\circ} 39^{\prime} 13^{\prime \prime} \mathrm{N}, 101^{\circ} 58^{\prime} 34^{\prime \prime} \mathrm{E}, 9.06 .2012$; all leg. I.A. Belousov, G.E. Davidian, I.I. Kabak \& A.E. Korolev; 1 o', 1 9 (ZMUM), Sichuan Prov., Jiulong County, SW of Wulaxixiang, $2800 \mathrm{~m}$ a.s.l., broadle- 


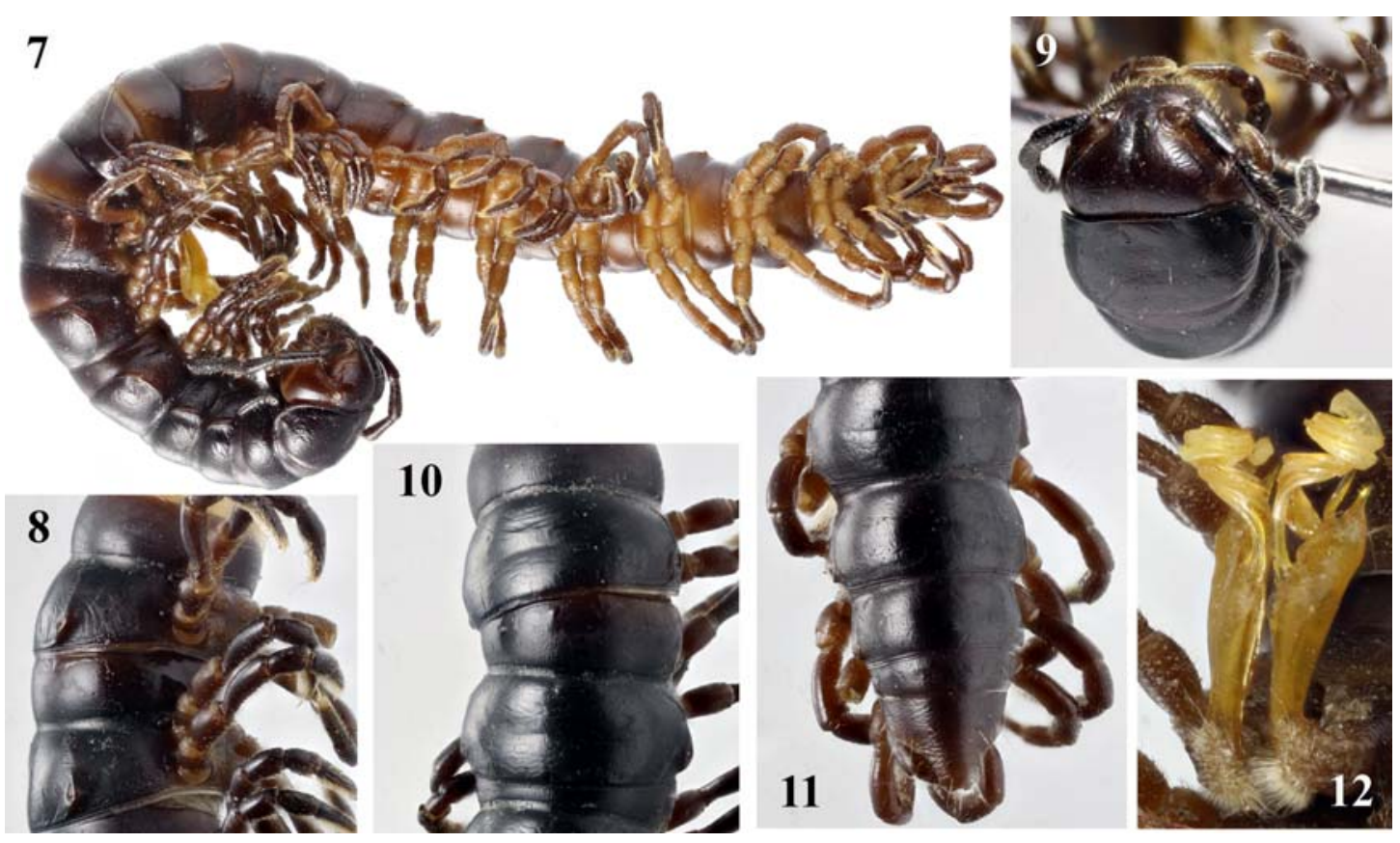

Figs 7-12. Inversispina erectispina Golovatch, 2012, $0^{7}$ from SW of Wulaxixiang: 7 - habitus, lateral and ventral; 8 \& $10-$ midbody segments, lateral and dorsal views, respectively; 9 - head and collum, front view; 11 — posterior part of body, dorsal; 12 gonopods, ventral. Photos taken by K. Makarov, not to scale.

Рис. 7-12. Inversispina erectispina Golovatch, 2012, о7 из юго-западнеe Wulaxixiang: 7 - общий вид, сбоку и снизу; 8 и $10-$ среднетуловищные сегменты, соответственно сбоку и сверху; 9 - голова и коллум, спереди; 11 — задняя часть тела, сверху; 12 гоноподы, снизу. Фото сделаны К. Макаровым, без масштаба.
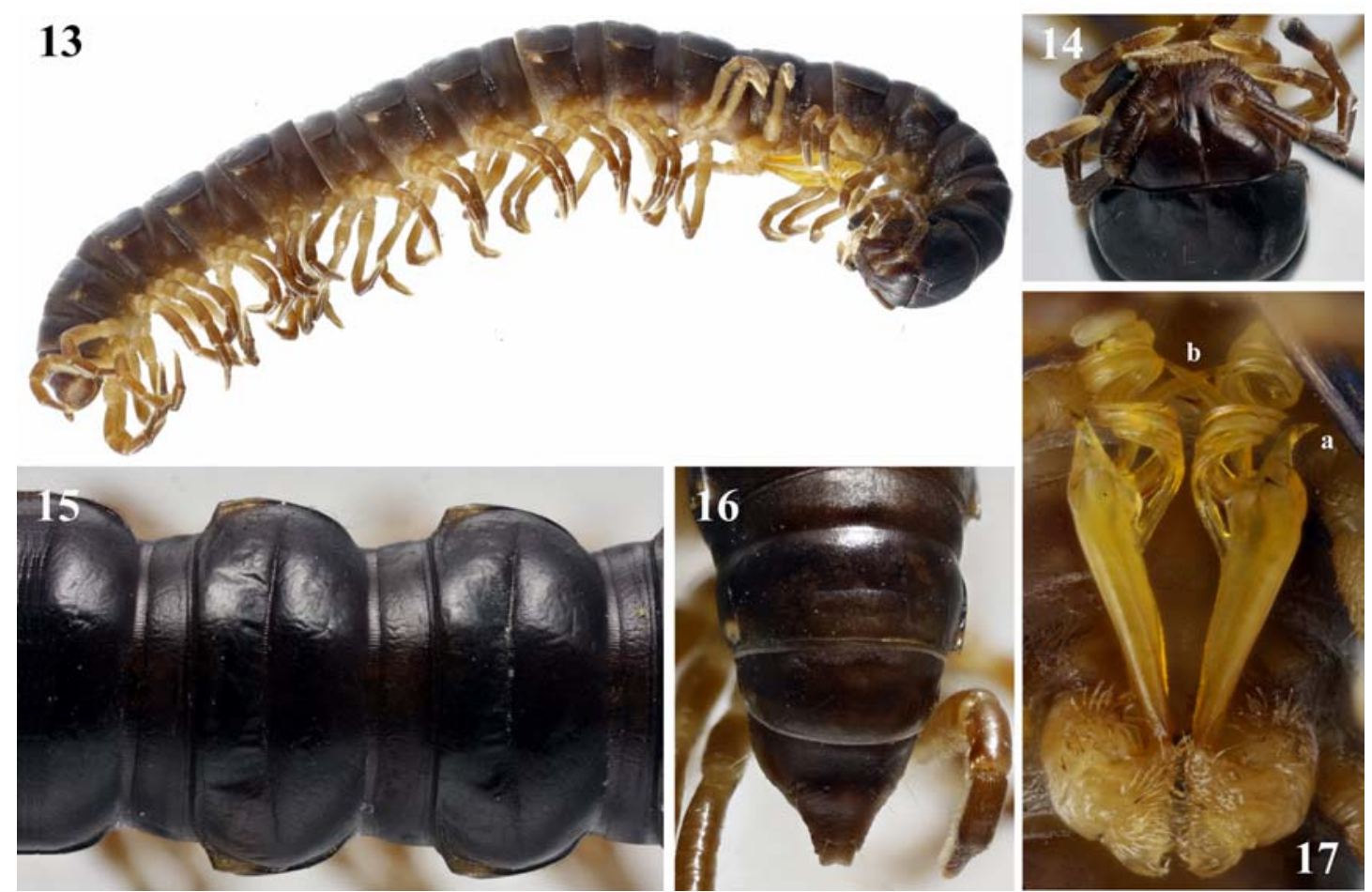

Figs 13-17. Inversispina erectispina Golovatch, 2012, $\mathrm{O}^{7}$ from NW of Mianning (2955 m a.s.1.): 13 - habitus, lateral; 14 - head and collum, front view; 15 - midbody segments, lateral; 16 - posterior body part, dorsal; 17 — gonopods, ventral. Photos taken by K. Makarov, not to scale.

Рис. 13-17. Inversispina erectispina Golovatch, 2012, О7 из северо-западнее Mianning (2955 м н.у.м.): 13 — общий вид, сбоку; 14 - голова и коллум, спереди; 15 - среднетуловищные сегменты, сверху; 16 - задняя часть тела, сверху; 17 - гоноподы, снизу. Фото сделаны К. Макаровым, без масштаба. 

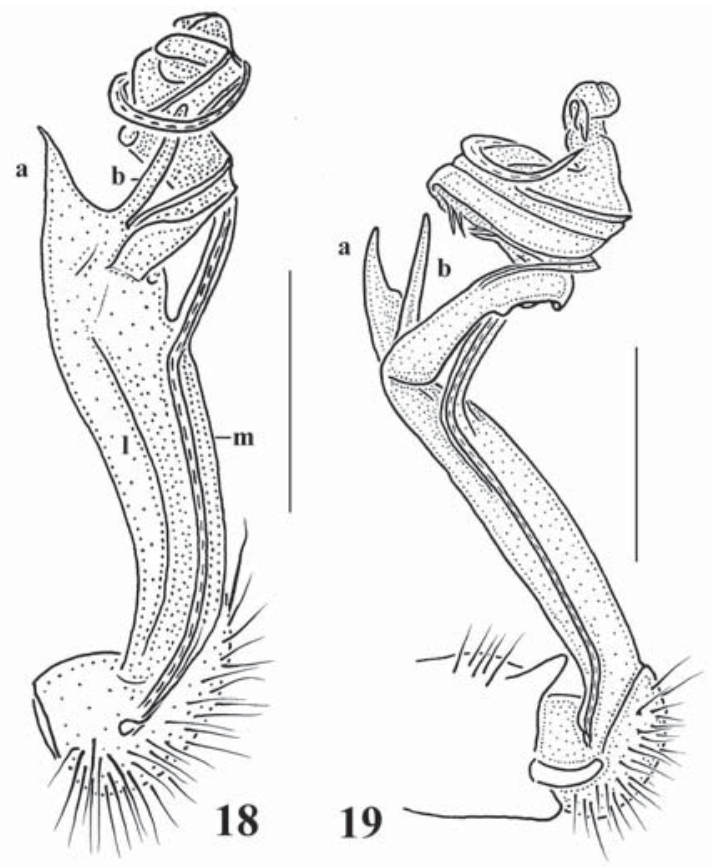

Figs 18 \& 19. Left gonopods of Inversispina erectispina Golovatch, 2012, $\sigma^{7}$ from SW of Wulaxixiang (18) and $\sigma^{7}$ from NW of Mianning (3895 m a.s.1.) (19). Scale bars: $1.0 \mathrm{~mm}$.

Рис. 18 и 19. Левые гоноподы Inversispina erectispina Golovatch, 2012, $\sigma^{7}$ из северо-западнее Wulaxixiang (18) и $\sigma^{7}$ из C3 Mianning (3895 м н.у.м.) (19). Масштаб 1,0 мм.

aved forest, $28^{\circ} 35^{\prime} 51^{\prime \prime} \mathrm{N}, 101^{\circ} 38^{\prime} 01^{\prime \prime} \mathrm{E}, 11.06 .2012 ; 2$ ○ (ZMUM), Sichuan Prov., Muli County, SW of Wulaxixiang, 4100 $\mathrm{m}$ a.s.1., broadleaved forest, $28^{\circ} 32^{\prime} 19^{\prime \prime} \mathrm{N}, 101^{\circ} 33^{\prime} 30^{\prime \prime} \mathrm{E}, 16.06 .2012$, all leg. I.A. Belousov \& G.E. Davidian.

REMARKS. The above near-topotypes are also high-montane, ranging between 2800 and $4100 \mathrm{~m}$ a.s.1., all previous encounters having been restricted to elevations exceeding $3200 \mathrm{~m}$ a.s.1. [Golovatch, 2012].

The new samples show evident variations in size and gonopod structure. Thus, specimens from somewhat lower elevations (ca $3000 \mathrm{~m}$ a.s.1.) tend to be larger and often a little paler than those from higher altitudes (4100 $\mathrm{m}$ a.s.1.): length up to 39-40 ( $\left.\mathrm{O}^{7}\right)$ or $42-$ $43 \mathrm{~mm}(+)$, width of midbody pro- and metazona up to 4.2 and $5.0\left(\bigcirc^{7}\right)$ or 4.5 and $5.2 \mathrm{~mm}(+)$, respectively, versus 32-35 $\left(\sigma^{7}\right), 2.5-2.6$ and $2.9-3.0 \mathrm{~mm}\left(\sigma^{7}\right)$, respectively; coloration uniformly brown to dark chocolate brown (Figs 7-11 \& 13-16). Golovatch [2012] also noted certain variation in size, albeit regardless of altitude: length $25-30 \mathrm{~mm}$ in both sexes, width of midbody pro- and metazona $2.4-2.7$ and 2.6-3.1 $\left(\sigma^{7}\right)$ or 3.0-3.5 and 3.5-4.0 $\mathrm{mm}(+)$, respectively. Variation concerns gonopod structure as well, this likely being microgeographic or even individual. Thus, the gonofemorite can have a clear-cut medial impression or gutter supporting the seminal groove at its bottom, this gutter being flanked by evident lobes (Fig. 18, 1 \& $\mathbf{m})$, whereas the distofemoral processes $\mathbf{a}$ and $\mathbf{b}$ are usually subequal in length (Figs 12, 18 \& 19), but sometimes a subflagelliform $\mathbf{b}$ is about twice as long as a (Fig. 17).
Gonobelus pentaspinus sp.n.

Figs 20-34.

HOLOTYPE $\sigma^{7}$ (ZMUM), China, Sichuan Prov., NW of Mianning, $2475 \mathrm{~m}$ a.s.1., $28^{\circ} 38^{\prime} 41^{\prime \prime} \mathrm{N}, 102^{\circ} 03^{\prime} 59^{\prime \prime} \mathrm{E}$, lower border of a broadleaved forest near village, 5.06.2012, leg. I.A. Belousov, G.E. Davidian, I.I. Kabak \& A.E. Korolev.

NAME. To emphasize five distinct spines becoming one (p) at the base of the solenophore.

DIAGNOSIS. Differs from both hitherto known congeners, G. sinensis Attems, 1936, from Yunnan Province, and G. martensi Golovatch, 2013, from Shaanxi Province, China [Golovatch, 2013], by the medium size (width ca $1.8 \mathrm{~mm}, \mathrm{O}^{7}$ ), by process $\mathbf{p}$ at the base of the solenophore being divided into five strong spines, opposed by a subtriangular lobe I (Figs 32-34), and by the sternal lobe between $O^{7}$ coxae 4 being remarkably high (Fig. 29).

DESCRIPTION. Length ca $23 \mathrm{~mm}$, width of midbody pro- and metazona 1.6 and $1.8 \mathrm{~mm}$, respectively. General coloration in alcohol uniformly brown to dark brown (Figs 20-24); tip of antennae, venter and proximal podomeres whitish, distal podomeres light brown.

Body submoniliform. In width, head $>$ segments 2 \& $5=16>3 \& 4>$ collum; body behind segment 16 gradually tapering towards telson. Clypeolabral region densely setose, vertigial region bare. Antennae long and very slender, reaching behind body segment 3 when stretched dorsally $\left(\mathrm{O}^{7}\right)$; antennomeres $2-5$ subequal, each only slightly longer than highest $6^{\text {th }}$. Paraterga very poorly developed (Figs 20-26), a small ventrolateral lobule in collum, small, invariably rounded, caudally subtruncate ridges never produced caudad behind tergal margin in following segments $2-19$, set low, mostly lying at about half of midbody height; slightly thinner and smaller in poreless segments compared to pore-bearing ones; calluses thin, delimited by a rather deep, arcuate sulcus only dorsally. Ozopores lateral, invisible from above, lying inside an ovoid groove slightly in front of caudal margin (Fig. 25). Body surface smooth and shining throughout. Axial line missing. Transverse metatergal sulcus thinner and incomplete in segments $2-4$ and 18 , deeper and longer, but still rather superficial, line-shaped and not reaching bases of paraterga in segments $5-17$, absent from $19^{\text {th }}$. Tergal setae rather short (ca $1 / 5^{\text {th }}$ of metatergal length), mostly abraded, arranged in a single transverse row of $1+1$ in front of sulcus, pattern barely traceable due to vague insertion points (Figs $25 \& 26$ ). Stricture dividing pro- and metazona rather broad, shallow, finely striolate dorsally. Pleurosternal carinae wanting. Epiproct (Figs 21, 24 \& 27) rather long, flattened dorsoventrally, subtruncate, subapical lateral papillae small. Hypoproct (Fig. 28) semi-circular, caudal 1+1 setae clearly separated, not borne on knobs.

Sternites without modifications, sparsely setose, cross-impressions modest, axial ones especially weak; a very high, linguiform, slender, laterally setose lamina between coxae 4 (Fig. 29). Legs very long and slender (Figs 20, 21, 23 \& 31), ca 2.3-2.4 times as long as 

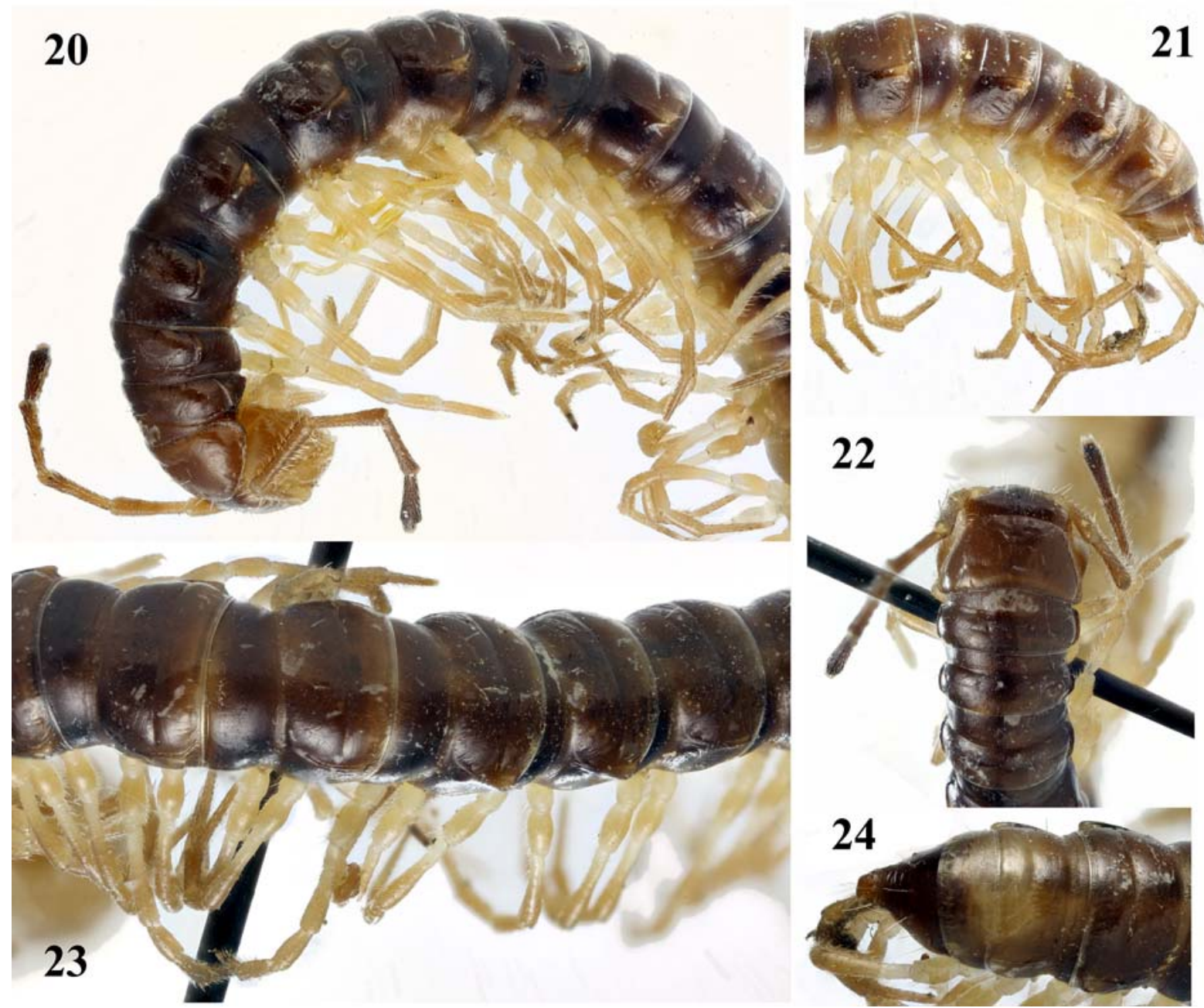

Figs 20-24. Gonobelus pentaspinus sp.n., holotype: 20 \& 22 - anterior part of body, lateral and dorsal views, respectively; 21 \& 24 posterior part of body, lateral and dorsal views, respectively; 23 - midbody segments, dorsal. Photos taken by K. Makarov, not to scale.

Рис. 20-24. Gonobelus pentaspinus sp.n., голотип: 20 и 22 - передняя часть тела, соответственно сбоку и сверху; 21 и 24 задняя часть тела, соответственно сбоку и сверху; 23 - среднетуловищные сегменты, сверху. Фото сделаны К. Макаровым, без масштаба.

midbody height $\left(\bigcirc^{7}\right)$; each prefemur 1 with a small, but evident ventral bulge/adenostyle (Fig. 30); all prefemora beset brush-like with longer setae ventrally (Fig. 31), tarsal brushes present until about legs of segment 15 , gradually thinning out thereafter.

Gonopods (Figs 32-34) fairly complex; coxite rather short, subcylindrical, with only a few setae distoventrally; prefemoral part as usual, densely setose, relatively short, $>2$ times shorther than acropodite; femorite rather long and very slender, nearly as long as solenophore or prefemorite; solenophore (sph) slender, clearly coiled, simple and ribbon-shaped, only a little shorter than a flagelliform solenomere; sph base with a palm-shaped ventral process (p) divided into five distinct spines and opposed by a large subtriangular lobe (I); seminal groove running entirely on mesal face of femorite before moving onto a free solenomere behind $\mathbf{p}$.

\section{Kronopolites semirugosus sp.n.}

Figs 35-44.

HOLOTYPE O $0^{7}$ (ZMUM), China, Sichuan Prov., NW of Mianning, $2955 \mathrm{~m}$ a s. $1 ., 28^{\circ} 39^{\prime} 13^{\prime \prime} \mathrm{N}, 101^{\circ} 58^{\prime} 34^{\prime \prime} \mathrm{E}$, broadleaved forest above village, 9.06.2012, leg. I.A. Belousov, G.E. Davidian, I.I. Kabak \& A.E. Korolev.

PARATYPES: 1 (ZMUM), same data, together with holotype; $1 \sigma^{\prime \prime}$ (ZMUM), same locality, $2155 \mathrm{~m}$ a.s.1., $28^{\circ} 38^{\prime} 28^{\prime \prime} \mathrm{N}$, $102^{\circ} 05^{\prime} 25^{\prime \prime} \mathrm{E}$, lower border of a broadleaved forest, 4.06.2012, leg. I.A. Belousov, G.E. Davidian, I.I. Kabak \& A.E. Korolev.

NAME. To emphasize the obvious similarities to K. rugosus Golovatch, 2013, from Yunnan, chiefly because of the likewise rugose/rugulose collum and following metaterga.

DIAGNOSIS. Differs from the geographically closest $K$. rugosus, the only other known congener among all eight described which shows clearly rugose/rugulose collum and following metaterga, by the much small- 


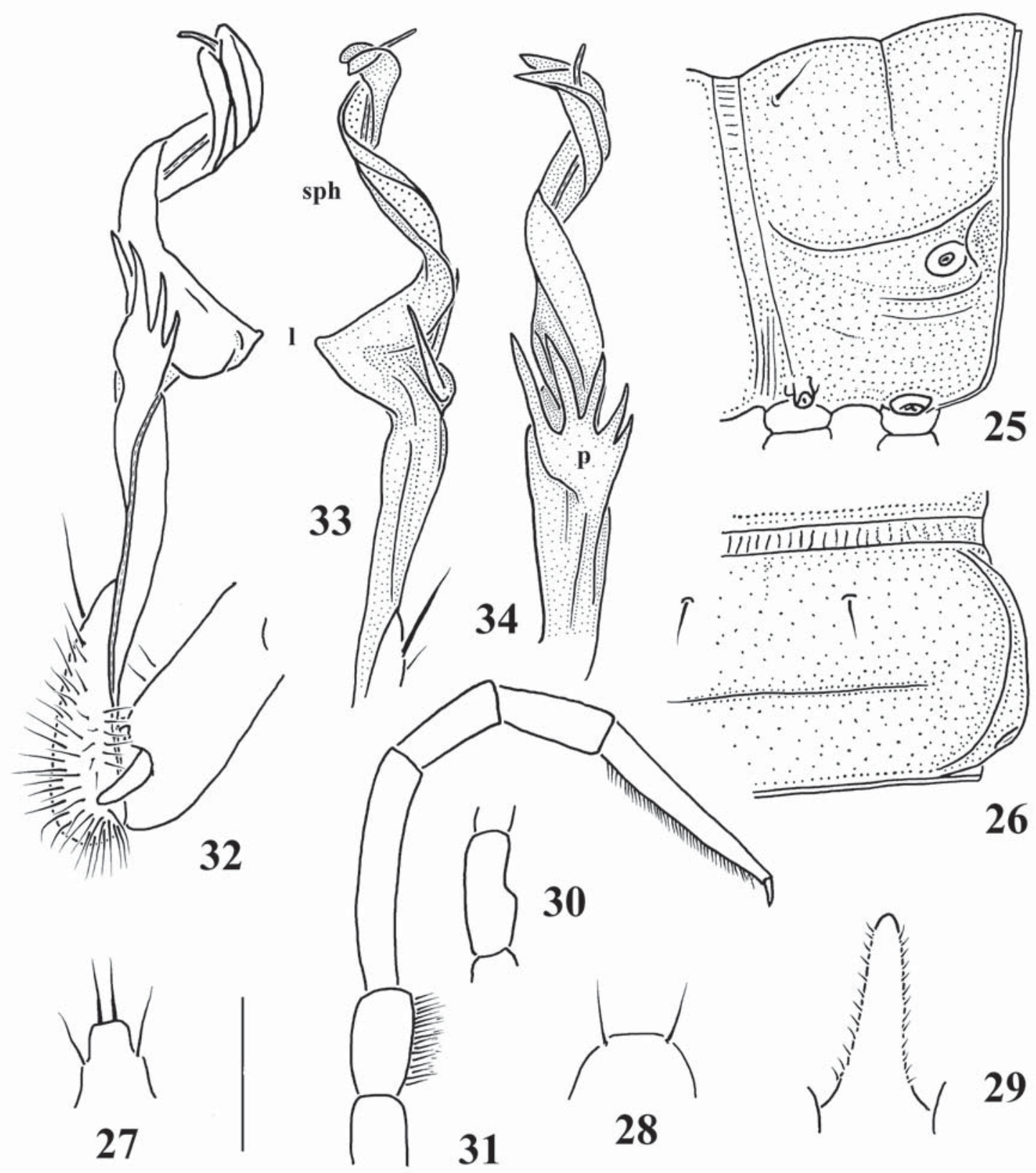

Figs 25-34. Gonobelus pentaspinus sp.n., holotype: $25 \& 26$ - body segment 10, lateral and dorsal views, respectively; 27 epiproct tip, dorsal; 28 - hypoproct, ventral; 29 - sternal lobe between coxae 4, caudoventral view; 30 - prefemur 1 , lateral; 31 midbody leg, lateral; 32-34 - right gonopod, mesal, lateral and ventral views, respectively. Scale bars: 0.7 (25-31) and $0.5 \mathrm{~mm}$ (32-34).

Pис. 25-34. Gonobelus pentaspinus sp.n., голотип: 25 и 26 - сегмент 10, соответственно сбоку и сверху; 27 - эпипрокт, сверху; 28 - гипопрокт, снизу; 29 - стернальная пластина между тазиками 4, вид одновременно снизу и сзади; 30 - предбедро 1 , сбоку; 31 - среднетуловищная нога, сбоку; 32-34 - правый гонопод, соответственно изнутри, сбоку и снизу. Масштаб 0,7 (25-31) и 0,5 мм (32-34).

er size (width up to $2.5 \mathrm{~mm}$ in $\bigcirc^{7}$ and $3.0 \mathrm{~mm}$ in the 9 of the new species, versus $3.9 \mathrm{~mm}$ in $\sigma^{7} K$. rugosus), the lack of an axial line, and the shape of the solenophore [Golovatch, 2013].

DESCRIPTION. Length ca 20 (holotype), 22 ( $\sigma^{7}$ paratype) or $27 \mathrm{~mm}$ ( 9 paratype), width of midbody pro- and metazona 1.8 and 2.2, 2.0 and 2.5 , or 2.7 and
$3.0 \mathrm{~mm}$, respectively. General coloration in alcohol uniformly blackish (Figs 35-37), sometimes head and ventrolateral regions of segments slightly lighter, dark brown; tip of antennae nearly pallid, legs and caudal halves of poriferous paraterga grey-brown.

In width, collum \& segments $5=17>$ head $=4>2=$ 3 ; body behind segment 17 gradually tapering towards 


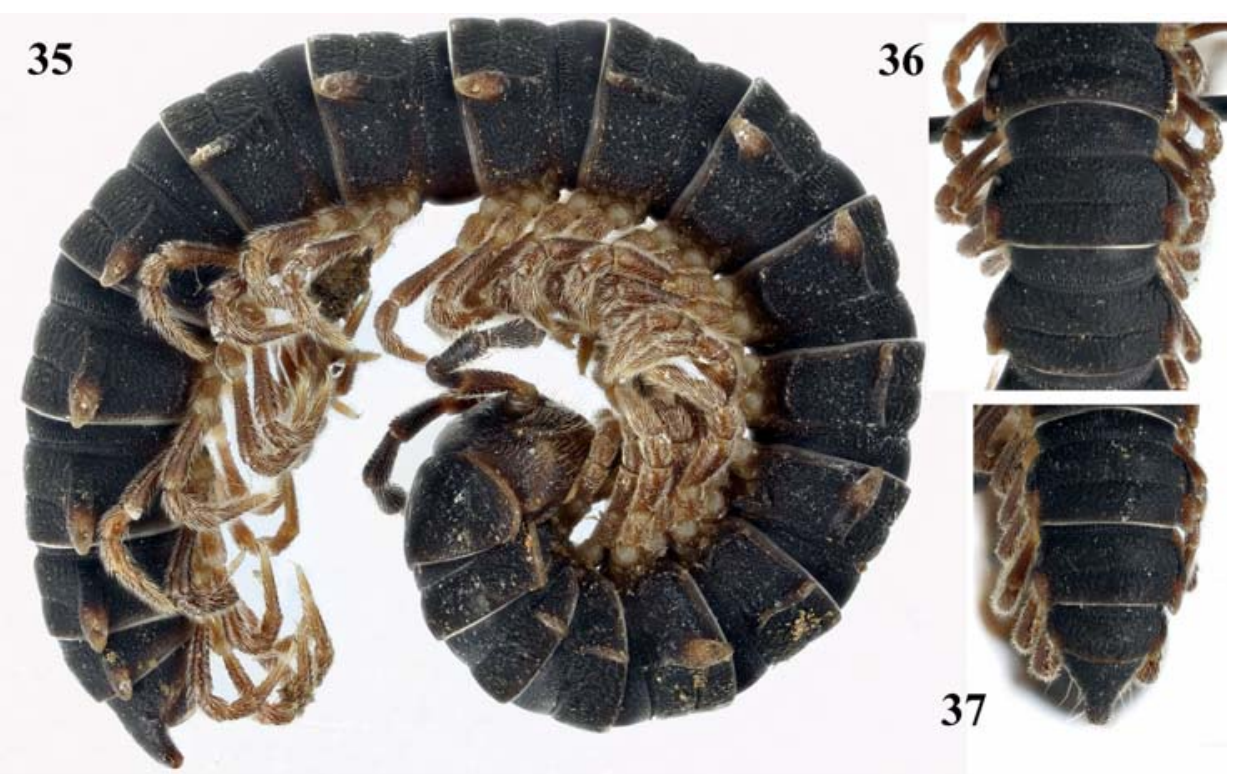

Figs 35-37. Kronopolites semirugosus sp.n., O paratype: 35 - habitus, lateral; 36 - midbody segments, dorsal; 37 - caudal body part, dorsal. Photos taken by K. Makarov, not to scale.

Рис. 35-37. Kronopolites semirugosus sp.n., паратип $0^{7}: 35$ - общий вид, сбоку; 36 - среднетуловищные сегменты, сверху; 37 задняя часть тела, сверху. Фото сделаны К. Макаровым, без масштаба.

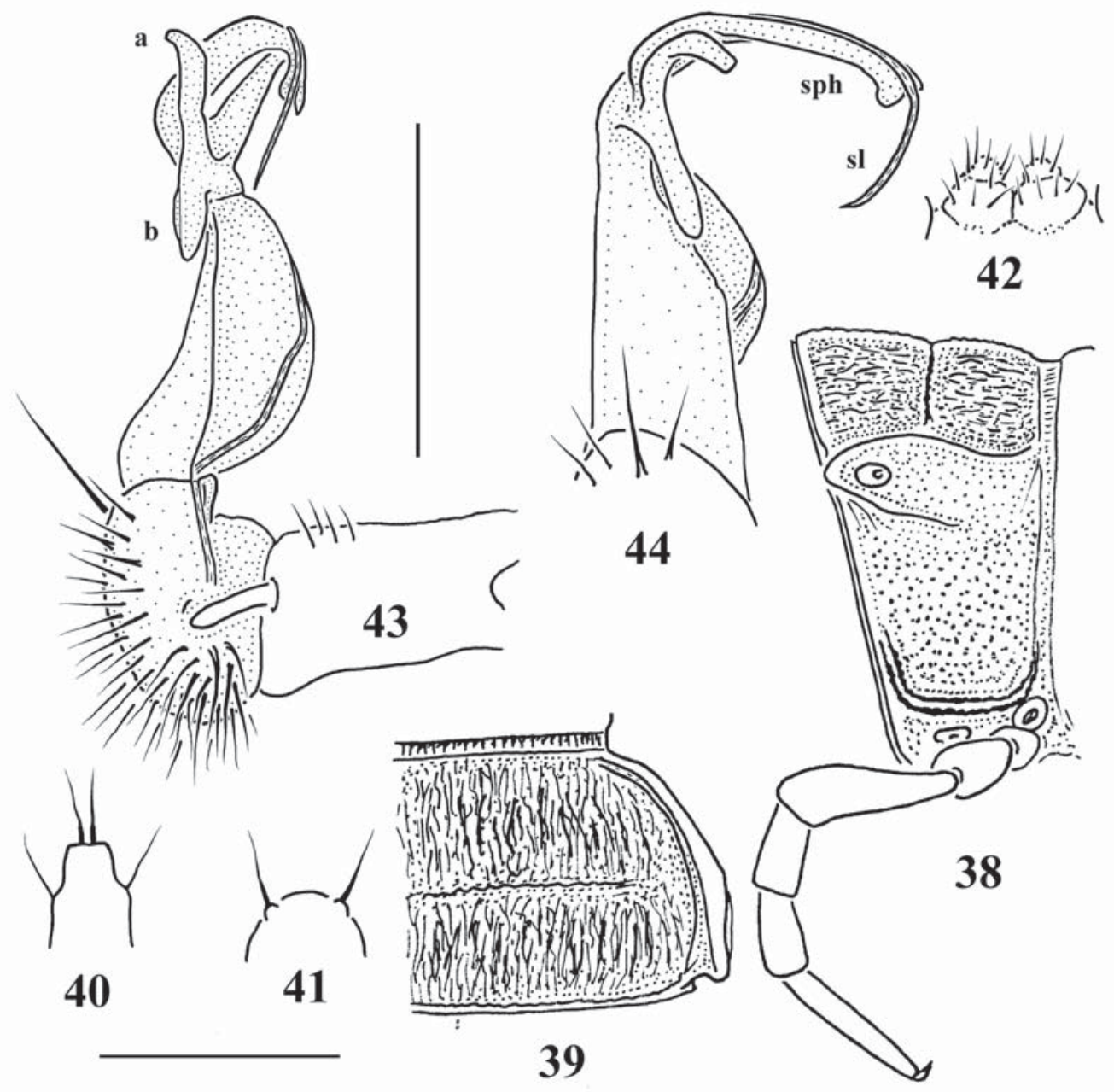

Figs 38-44. Kronopolites semirugosus sp.n., O' paratype: 38 \& 39 - body segment 10, lateral and dorsal views, respectively; 40 epiproct tip, dorsal; 41 - hypoproct, ventral; 42 - sternal tubercles between coxae 4, caudoventral view; 43 \& 44 - right gonopod, mesal and ventral views, respectively. Scale bars: 1.0 (38-42) and $0.5 \mathrm{~mm}(43 \& 44)$.

Рис. 38-44. Kronopolites semirugosus sp.n., паратип О7: 38 и 39 - сегмент 10, соответственно сбоку и сверху; $40-$ эпипрокт, сверху; 41 - гипопрокт, снизу; 42 — стернальные бугорки между тазиками 4, вид одновременно снизу и сзади; 43 и 44 - правый гонопод, соответственно изнутри и снизу. Масштаб 1,0 (38-42) и 0,5 мм (43 и 44). 

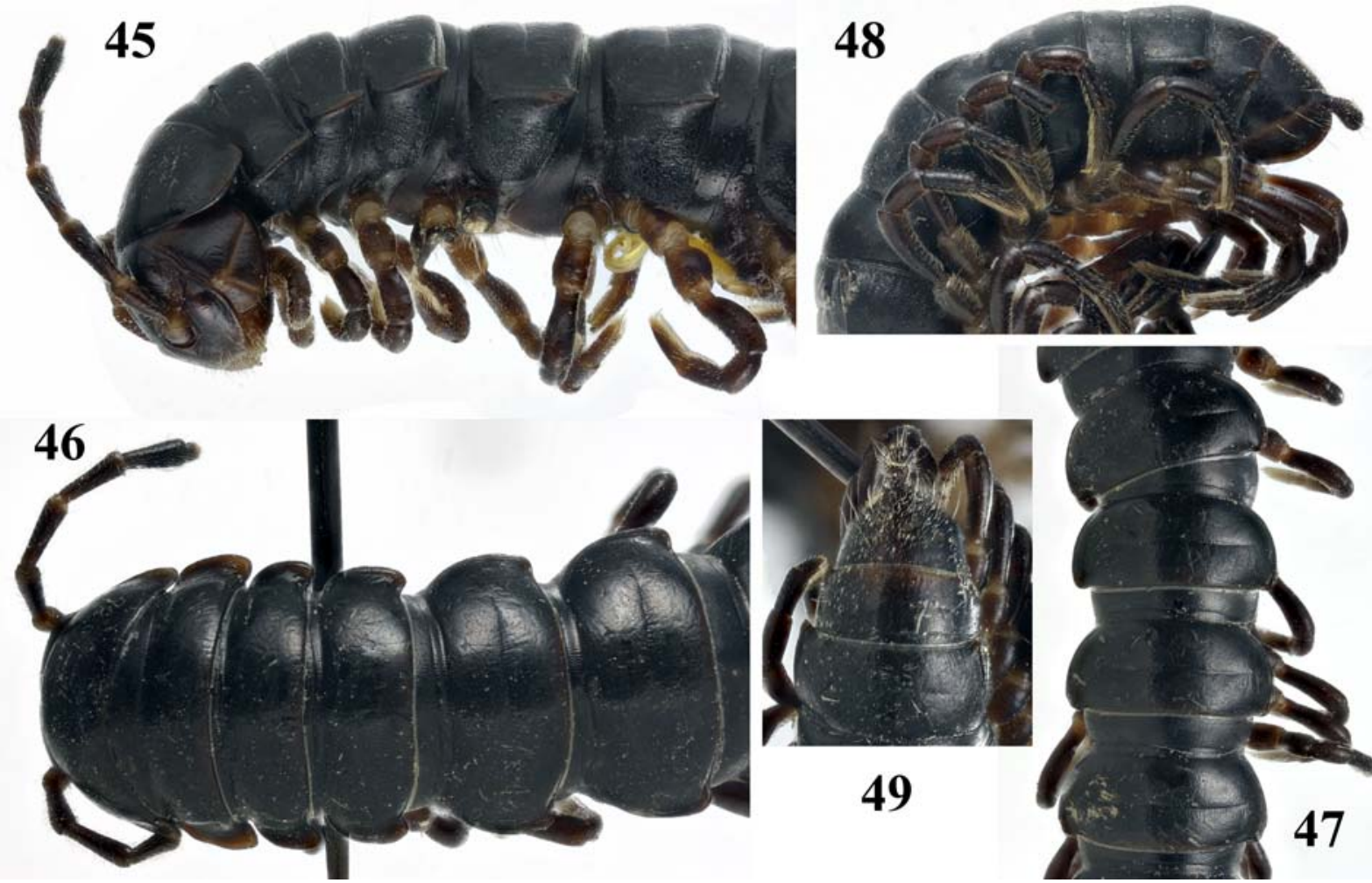

Figs 45-49. Hedinomorpha nigra sp.n., holotype: 45 \& 46 - anterior body part, lateral and dorsal views, respectively; 47 - midbody segments, dorsal; 48 \& 49 - caudal body part, lateral and dorsal views, respectively. Photos taken by K. Makarov, not to scale.

Рис. 45-49. Hedinomorpha nigra sp.n., голотип: 45 и 46 - передняя часть тела, соответственно сбоку и сверху; 47 среднетуловищные сегменты, сверху; 48 и 49 - задняя часть тела, соответственно сбоку и сверху. Фото сделаны К. Макаровым, без масштаба.

telson. Clypeolabral region densely setose, vertigial region bare. Antennae rather short and slightly clavate, reaching behind body segment $2\left(O^{7}\right)$ or its midway $(+)$ when stretched dorsally; antennomeres $2-5$ subequal, each only slightly longer than highest $6^{\text {th }}$. Paraterga poorly $\left(\sigma^{7}\right)$ (Figs 35-39) to very poorly developed $(+)$, a large, broadly rounded, ventrolateral lobe in collum, modest, caudally invariably rounded ridges usually only reaching the caudal tergal margin, extended caudad behind the latter only in a few $O$ poriferous segments in hind body half, best so on $17^{\text {th }}$ and $18^{\text {th }}\left(\sigma^{\text {th }}\right)$, set rather high, mostly lying at about $1 / 3$ of midbody height; slightly thinner and smaller in poreless segments compared to pore-bearing ones; calluses thin, delimited by a rather deep, arcuate sulcus both dorsally and ventrally $\left(O^{7}\right)$ or only dosally $(+)$. Ozopores lateral, invisible from above, lying inside an ovoid groove slightly in front of caudal margin (Figs $35 \& 38$ ). Body surface dull, prozona finely shagreened, metazona rugulose to rugose dorsally, rather coarsely granulate below paraterga. Axial line missing. Transverse metatergal sulcus thin, line-shaped, complete and reaching bases of paraterga in segments $5-18$, absent from $19^{\text {th }}$. Tergal setae wanting, pattern untraceable. Stricture dividing pro- and metazona rather broad, shallow, finely stri- olate dorsally and microgranulate below paraterga. Pleurosternal carinae well-developed, arcuate and granulate ridges with a caudal denticle until segment 9, increasingly poorly developed crests traceable until segment 17 , faint bulges in segment $18\left(\sigma^{7}\right)$, or low ridges traceable until segment 13 , low bulges in segments 14 and 15 (+). Epiproct (Figs $37 \&$ 40) long, flattened dorsoventrally, subtruncate, subapical lateral papillae small. Hypoproct (Fig. 41) semi-circular, caudal 1+1 setae clearly separated, borne on minute knobs.

Sternites without modifications, densely setose, crossimpressions modest, axial impressions especially weak; two low, setose, medially contiguous tubercles between coxae 4 (Fig. 42). Legs very densely setose, rather long and slender, with neither adenostyles nor dorsally bulged prefemora, 1.3-1.4 $\left(\sigma^{7}\right)$ times or about as long as midbody height ( $\left.{ }^{\circ}\right)$; $\sigma^{\top}$ tarsal brushes present until about midbody legs, gradually thinning out thereafter.

Gonopods (Figs 43 \& 44) rather complex; coxite long, subcylindrical, with only a few setae distoventrally; prefemoral part as usual, densely setose, relatively short, about half as long as acropodite; femorite rather short and broad, with a mesal groove; solenophore (sph) directed mesad, slender, simple and ribbon-shaped, considerably shorter than a flagelliform 

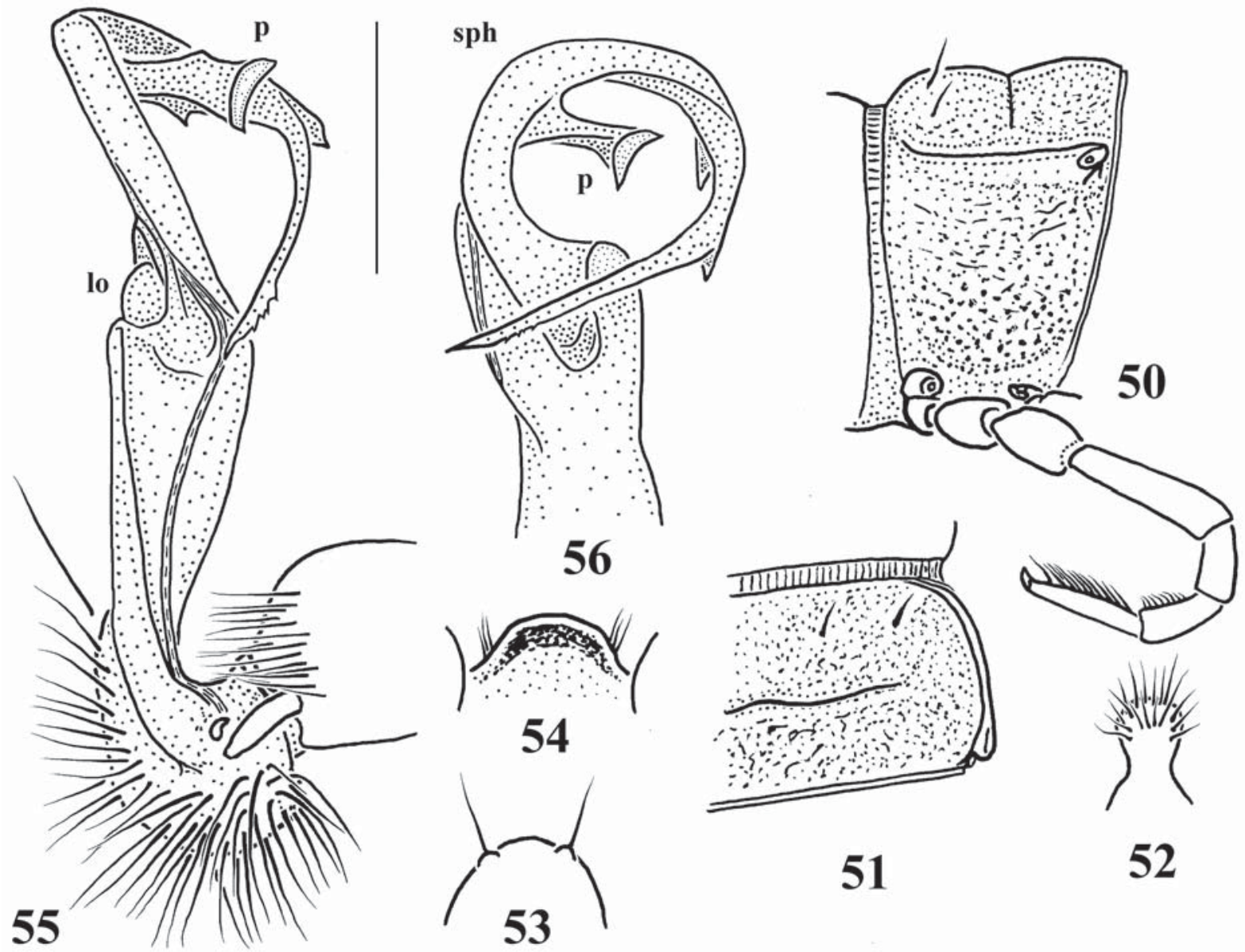

Figs 50-56. Hedinomorpha nigra sp.n., holotype: $50 \& 51$ - body segment 10, lateral and dorsal views, respectively; 52 - epiproct tip, dorsal; 53 - hypoproct, ventral; 54 - sternal lobe between coxae 4, caudoventral view; 55 \& 56 - right gonopod, mesal and dorsal views, respectively. Scale bars: $1.5(50-54)$ and $0.5 \mathrm{~mm}(55 \& 56)$.

Рис. 50-56. Hedinomorpha nigra sp.n., голотип: 50 и 51 - сегмент 10, соответственно сбоку и сверху; 52 - эпипрокт, сверху; 53 - гипопрокт, снизу; 54 - стернальная пластина между тазиками 4 , вид одновременно снизу и сзади; 55 и 56 - правый гонопод, соответственно изнутри и сверху. Масштаб 1,5 (50-54) и 0,5 мм (55 и 56).

solenomere (sl); base of sph with two subequal, ribbon-shaped, mesal processes (a and $\mathbf{b})$.

\section{Hedinomorpha nigra sp.n.}

Figs 45-56.

HOLOTYPE $0^{7}$ (ZMUM), China, Sichuan Prov., Jiuzhaigou County, N of Dajisi, $3530-4000 \mathrm{~m}$ a.s.1., $33^{\circ} 22^{\prime} 43^{\prime \prime} \mathrm{N}-33^{\circ} 23^{\prime} 20^{\prime \prime} \mathrm{N}$ $103^{\circ} 48^{\prime} 26^{\prime \prime} \mathrm{E}-103^{\circ} 49^{\prime} 01^{\prime \prime} \mathrm{E}$, from upper timberline of a broadleaved forest through subalpine to lower alpine meadows, 21.06.2012, leg. I.A. Belousov, G.E. Davidian, I.I. Kabak \& A.E. Korolev.

NAME. To emphasize the uniformly black coloration.

DIAGNOSIS. Differs from both known congeners showing a similarly clavate epiproct, i.e. H. biramipedicula Zhang \& Tang, 1985 and H. hummeli Verhoeff, 1933, in the peculiar, ribbon-shaped, distomesally strongly curved and apically subtruncate lateral process at the base of the solenophore [Golovatch, 2012, 2013]. See also Key below.

DESCRIPTION. Length ca $28 \mathrm{~mm}$, width of midbody pro- and metazona 2.5 and $3.0 \mathrm{~mm}$, respectively.
General coloration in alcohol uniformly blackish (Figs 45-49), only head, venter and legs dark brown, tip of antennae whitish.

In width, head $<$ collum $=$ segments $3 \& 4<$ segments $2=5-16$; thereafter body gradually tapering towards telson. Clypeolabral region densely setose, vertigial region bare. Antennae long and slender, reaching behind body segment 2 when stretched dorsally $\left(O^{7}\right)$; antennomeres $2-6$ subequal in length, $6^{\text {th }}$ highest. Paraterga poorly developed (Figs 45-51), always thin and at least somewhat declined ventrad; a small ventrolateral lobule in collum, small, caudally invariably rounded ridges only slightly produced caudad behind tergal margin in following segments $2,3,15-18$; set rather high, mostly lying at about $1 / 3$ of midbody height; only slightly thinner in poreless segments compared to pore-bearing ones; calluses thin, delimited by a rather deep, arcuate sulcus only dorsally. Ozopores lateral, invisible from above, lying inside an ovoid groove slightly in front of caudal margin (Figs $45 \& 50$ ). Body surface smooth and shining, prozona finely shagreened, 


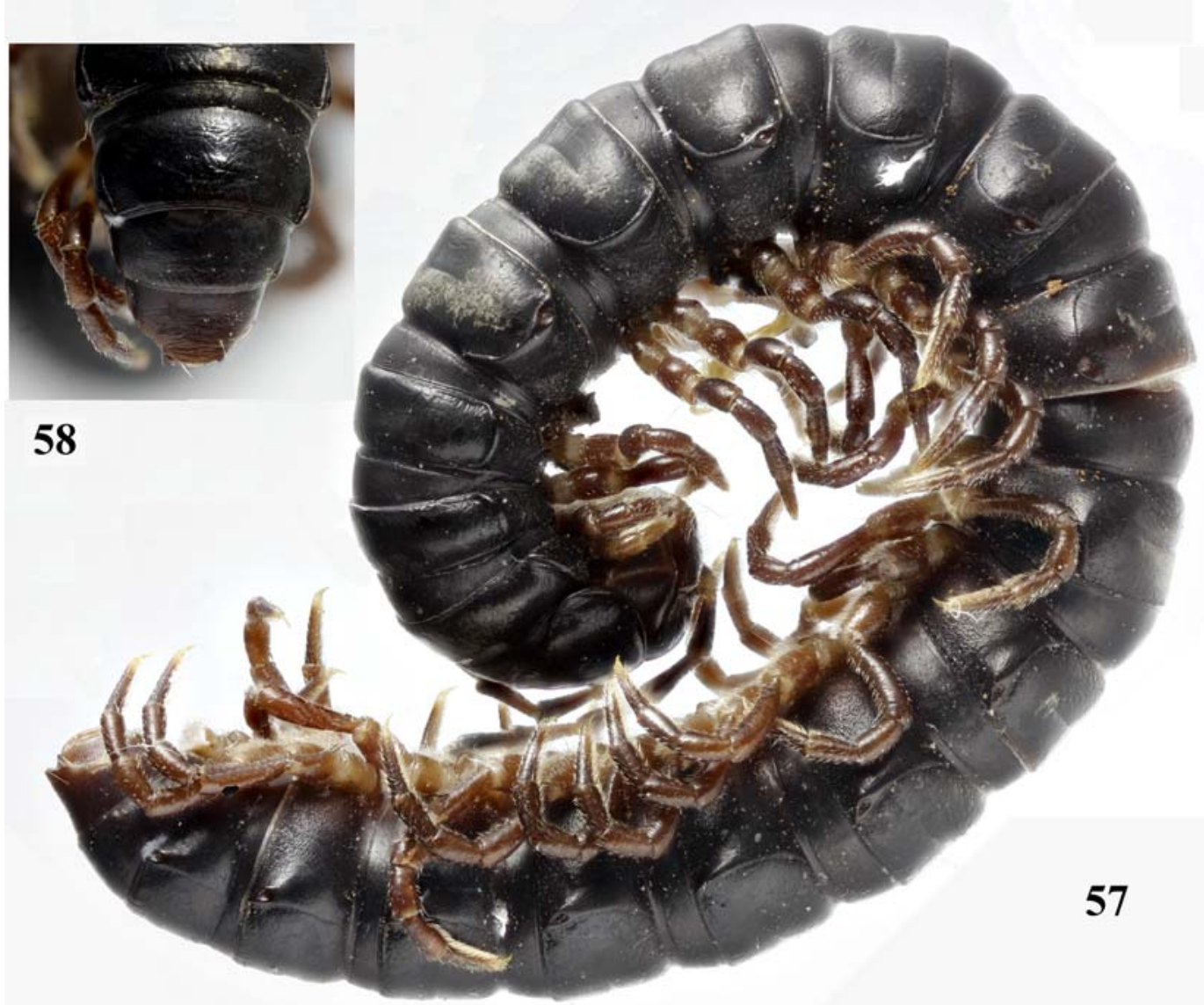

Figs 57 \& 58. Hedinomorpha subnigra sp.n., holotype: 57 - habitus, lateral; 58 - caudal body part, dorsal. Photos taken by K. Makarov, not to scale.

Рис. 57 и 58. Hedinomorpha subnigra sp.n., голотип: 57 - общий вид, сбоку; 58 - задняя часть тела, сверху. Фото сделаны К. Макаровым, без масштаба.

metazona smooth, only in places very faintly rugulose dorsally, microgranulate below paraterga. Axial line missing. Transverse metatergal sulcus thinner and incomplete in segment 18 , deeper and longer, line-shaped and reaching bases of paraterga in segments 5-17, absent from $19^{\text {th }}$. Tergal setae rather short (ca $1 / 5^{\text {th }}$ of metatergal length), mostly abraded, arranged in a single transverse row of $2+2$ in front of sulcus, pattern barely traceable due to vague insertion points (Figs 50 \& 51). Stricture dividing pro- and metazona rather narrow, finely striolate dorsally down to below paraterga. Pleurosternal carinae increasingly evident ridges until segment 5 , very strong, arcuated and microgranulate lobes with a rounded caudal tooth in segments 6 and 7 , then abruptly small ridges with traces of a caudal tooth until segment 9 (Fig. 45), thereafter increasingly reduced bulges in several following segments (Fig. 50). Epiproct (Figs 48, 49 \& 52) long, densely pubescent dorsally, distal half setose and clearly clavate. Hypoproct (Fig. 53) semi-circular, caudal $1+1$ setae clearly separated, borne on minute knobs.

Sternites without modifications, densely setose, crossimpressions modest, axial impression especially weak; a rather high, regularly rounded, laterally setose lamina between coxae 4 (Fig. 54), two paramedian bunches of long setae on next sternum. Legs long, rather slender (Figs 45-50), probably slightly incrassate compared to +, ca 1.4-1.5 times as long as midbody height $\left(O^{7}\right)$; prefemora very slightly bulged dorsally (Fig. 50); all prefemora beset brush-like with longer setae ventrally, tibial and tarsal brushes present until last two leg-pairs.

Gonopods (Figs 55 \& 56) fairly complex; coxite rather short, subcylindrical, setose distoventrally; prefemoral part as usual, densely setose, about 4 times shorter than acropodite, but only a little shorter than a distally enlarged femorite; the latter much shorter than a clearly coiled, simple, acuminate and ribbon-shaped solenophore (sph) which is about as long as a flagelliform solenomere; base of $\mathbf{s p h}$ with a ventral lobule (lo) and a strong, subtruncate, lateral, distomedially unciform process (p), whereas distal half with two small teeth, apex finely serrate.

\section{Hedinomorpha subnigra sp.n.}

Figs 57-64.

HOLOTYPE O (ZMUM), China, Yunnan Prov., W of Lake Lugu, $3910 \mathrm{~m}$ a.s.1., $27^{\circ} 41^{\prime} 57^{\prime \prime} \mathrm{N}, 100^{\circ} 34^{\prime} 06^{\prime \prime} \mathrm{E}$, upper timberline of a broadleaved forest with dry forest edges and low bushes, 28.05.2012, leg. I.A. Belousov, G.E. Davidian, I.I. Kabak \& A.E. Korolev. 


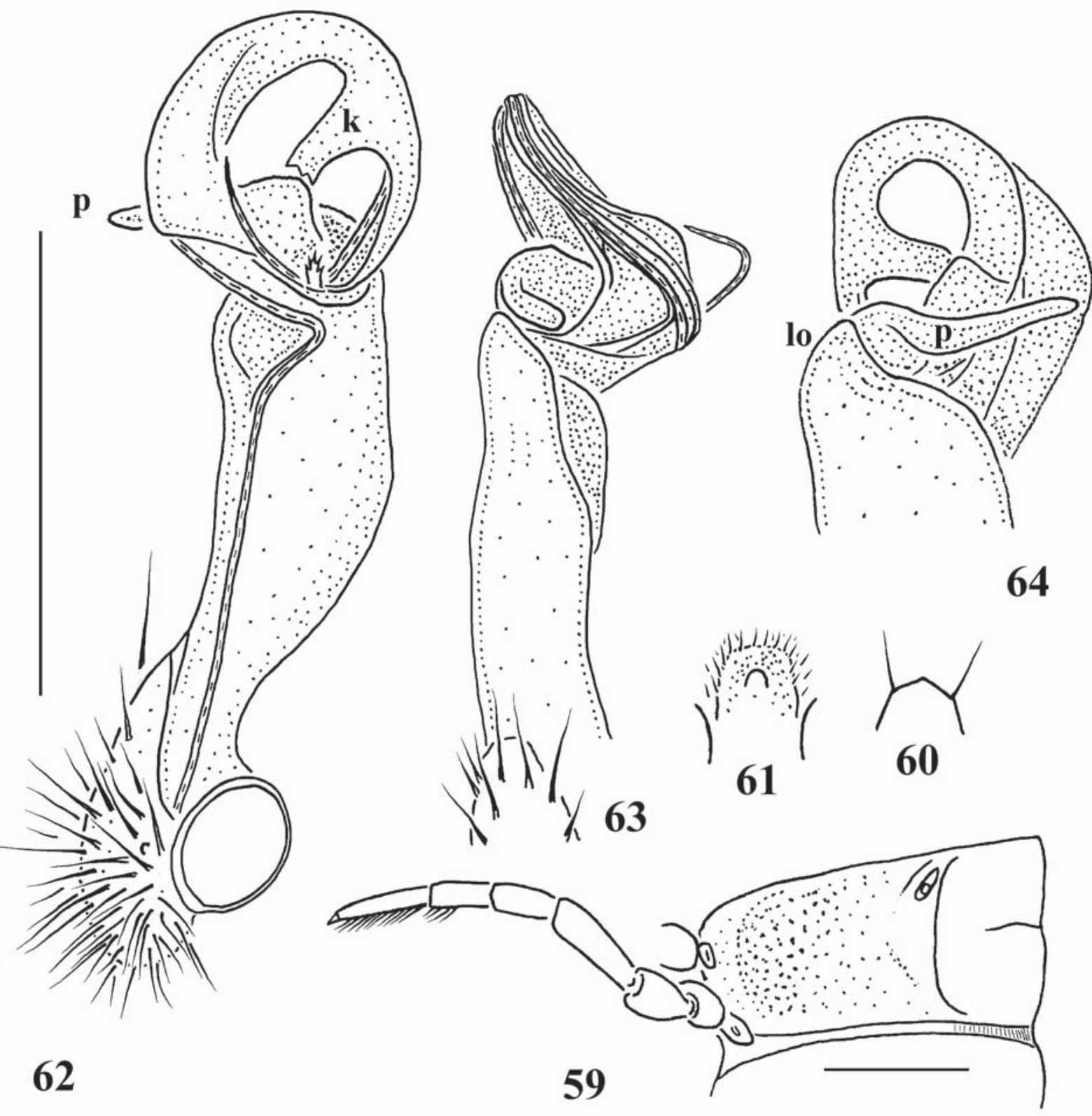

Figs 59-64. Hedinomorpha subnigra sp.n., holotype: 59 - body segment 10, lateral; 60 - hypoproct, ventral; 61 - sternal lobe between coxae 4, caudoventral view; 62-64 — right gonopod, mesal, ventral and lateral views, respectively. Scale bars: $1.0 \mathrm{~mm}$.

Рис. 59-64. Hedinomorpha subnigra sp.n., голотип: 59 - сегмент 10, сбоку; 60 - гипопрокт, снизу; 61 - стернальная пластина между тазиками 4, вид одновременно снизу и сзади; 62-64 - правый гонопод, соответственно изнутри, снизу и сбоку. Масштаб 1,0 мм.

NAME. To emphasize the similarities to the previous congener.

DIAGNOSIS. Differs from all known species of the genus by the peculiar, finger-shaped lateral process at the base of the solenophore [Golovatch, 2012, 2013]. See also Key below.

DESCRIPTION. Length ca $27 \mathrm{~mm}$, width of midbody pro- and metazona 2.5 and $3.0 \mathrm{~mm}$, respectively. General coloration in alcohol uniformly blackish (Figs $57 \& 58$ ), much like in $H$. nigra sp.n. lows.
Epiproct broken off (Figs 57 \& 58), obviously normal, non-clavate, because its base is devoid of dense pilosity, being supplied instead with only $1+1$ long setae. Only paraterga 2 very faintly protruding behind rear tergal margin (Fig. 57). Pleurosternal carinae absent already from segment 8 . Transverse sulcus on metaterga not reaching bases of paraterga (Fig. 59). Tergal setae missing, pattern untraceable. Hypoproct pentagonal (Fig. 60). Sternal lobe between coxae 4 linguiform, densely setose throughout, with a small midway knob on caudal face (Fig. 61). Legs a little shorter (Figs 57 \& 59). 


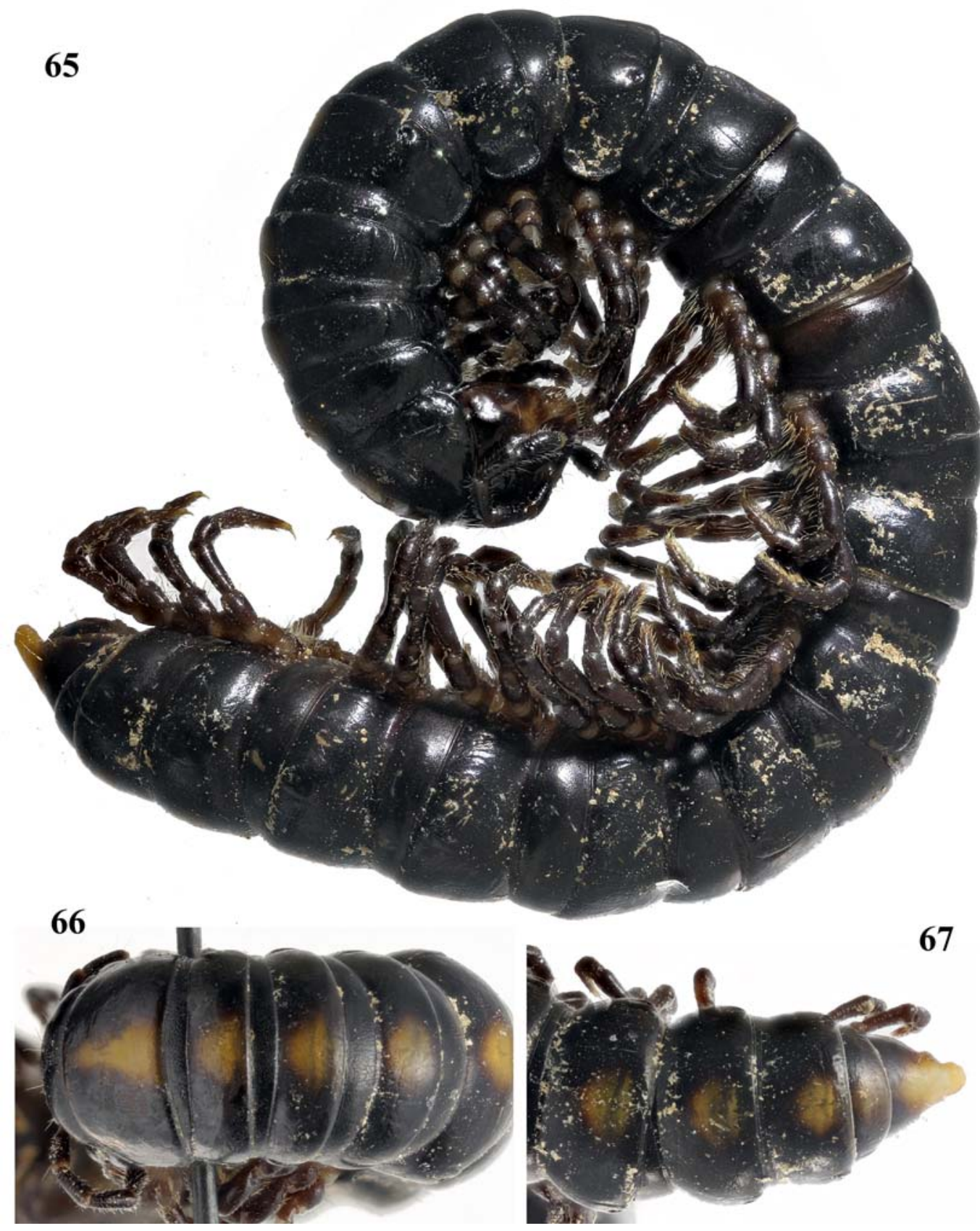

Figs 65-67. Sigipinius grahami Hoffman, 1961, $0^{7}$ from SW of Tonghua: 65 - habitus, lateral; 66 \& 67 — anterior and caudal body parts, respectively, dorsal. Photos taken by K. Makarov, not to scale.

Рис. 65-67. Sigipinius grahami Hoffman, 1961, О7 из юго-западнее Tonghua: 65 - общий вид, сбоку; 66 и 67 - соответственно передняя и задняя части тела, сверху. Фото сделаны К. Макаровым, без масштаба.

Gonopods (Figs 62-65) much like in H. nigra sp.n., but distofemoral lobule (lo) much smaller, process (p) at base of solenophore finger-shaped, rounded, directed ventrad, midway tooth (k) much stronger and subtruncate; solenophore slightly shorter than solenomere, fringed apically.

REMARKS. The genus Hedinomorpha Verhoeff, 1933 has hitherto been known to encompass only six valid species: H. hummelii Verhoeff, 1933 (the type species, with two varieties), from the southern part of Gansu Province, central China; H. bucharensis (Lohmander, 1933), from Tajikistan, Central Asia, H. biramipedicula Zhang et Tang, 1985, from Qinling Mountains, lying across the southern part of Shanxi Province, central China, H. jeekeli (Golovatch, 2009), from Shaanxi Province, central China, H. circofera Golovatch, 2013, from Qinghai Province, west-central China, and $H$. reducta Golovatch, 2012, from Sichuan 


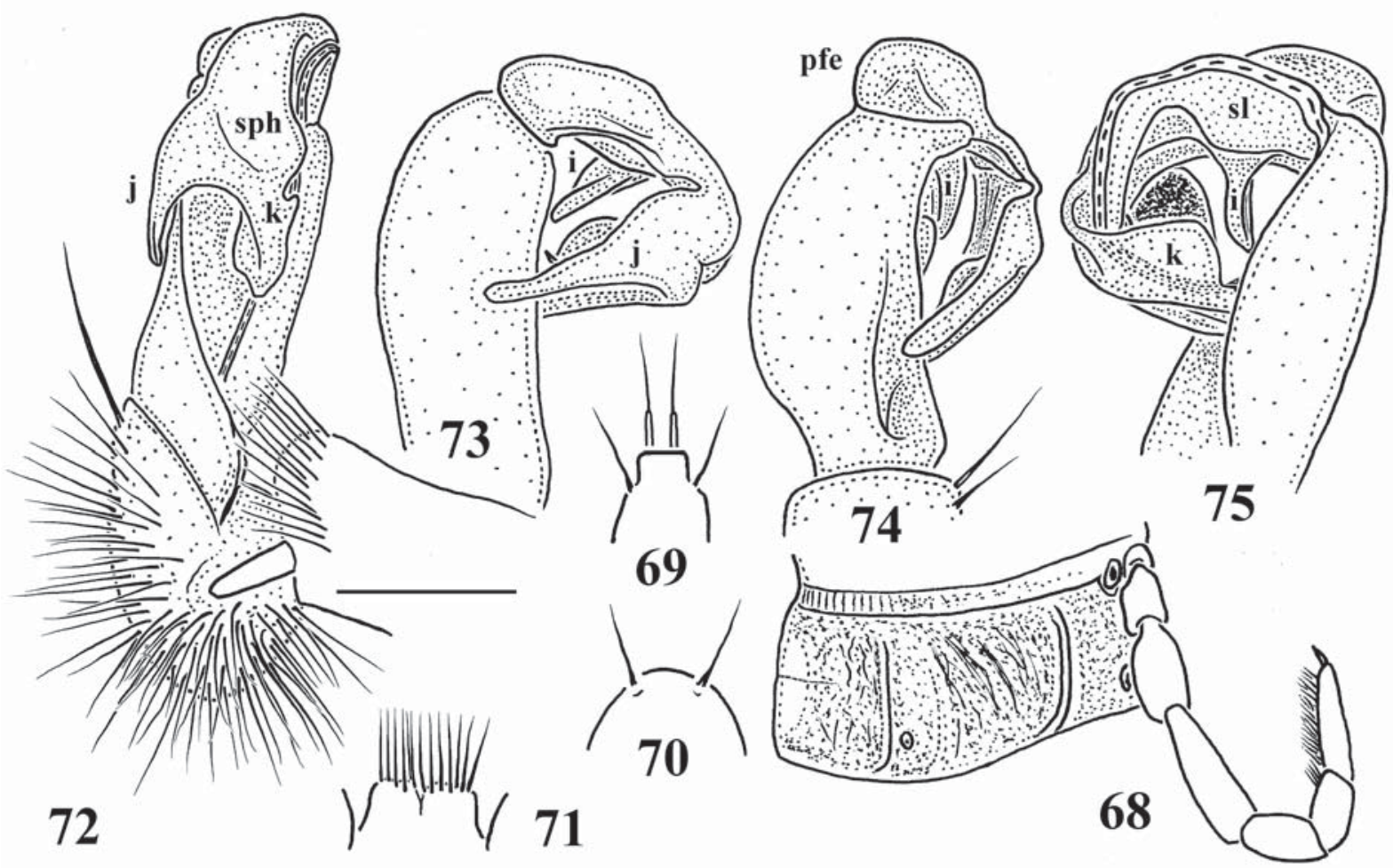

Figs 68-75. Sigipinius grahami Hoffman, 1961, ơ from SW of Tonghua: 68 - body segment 10, lateral; 69 - epiproct tip, dorsal; 70 - hypoproct, ventral; 71 - sternal lobe between coxae 4, caudoventral view; 72-75 - right gonopod, mesal, ventral, ventrolateral and dorsal views, respectively. Scale bar: $1.0(68-71)$ and $0.35 \mathrm{~mm}(72-75)$.

Рис. 68-75. Sigipinius grahami Hoffman, 1961, О из юго-западнее Tonghua: 68 - сегмент 10, сбоку; 69 - эпипрокт, сверху; 70 - гипопрокт, снизу; 71 - стернальная пластина между тазиками 4, вид одновременно снизу и сзади; 72-75 - правый гонопод, соответственно изнутри, снизу, одновременно снизу и сбоку, а также сверху. Масштаб 1,0 (68-71) и 0,35 мм (72-75).

Province, southern China [Verhoeff, 1933; Zhang \& Tang, 1985; Jeekel, 1988; Golovatch, 2009, 2012, 2013].

The following key can be proposed to separate all eight presently known species of Hedinomorpha Verhoeff, 1933.

1(2) Adults larger: body width $\geq 3.5 \mathrm{~mm}$. Paraterga especially poorly developed, low bulges to nearly missing. Gonopod femorite particularly slender, devoid of a mesal groove, lateral process at base of an acuminate solenophore unciform. Sichuan, China H. reducta

2(1) Adults smaller: body width $\leq 3.0 \mathrm{~mm}$. Paraterga keelshaped, at least some in caudal body third usually extending behind rear tergal margin. Gonopod femorite far less slender, a vague mesal impression often present, lateral process at base of a more usually non-acuminate solenophore shorter or much longer, not unciform .... 3

3(10) Epiproct normal, flattened dorsoventrally, not clavate

4(5) Solenophore subtruncate. Lateral process at base of solenophore a short, slender, subtruncate lobe. Tajiki$\operatorname{stan}$.

H. bucharensis

5(4) Solenophore subacuminate. Lateral process at base of solenophore either a short, subtriangular tooth or a long, slender, rounded finger. China

6(7) Lateral process (p) at base of solenophore a long rounded finger (Figs 62-64). ................. H. subnigra sp.n.

7(6) Lateral process at base of solenophore sharp
8(9) Lateral process at base of solenophore a short subtriangular tooth. ................................................. H. jeekeli

9(8) Lateral process at base of solenophore a long ribbon ....

H. circofera

10(3) Epiproct club-shaped (Figs 48, 49 \& 52) 11

11(12) Lateral process at base of solenophore a long ribbon (Figs $55 \& 56$ ). H. nigra sp.n.

12(11) Lateral process at base of solenophore different ....... .. 13

13(14) Lateral process at base of solenophore clearly bifid H. biramipedicula

14(13) Lateral process at base of solenophore subtruncate ... H. hummelii

\section{Sigipinius grahami Hoffman, 1961}

Figs $65-78$.

MATERIAL. $1 \sigma^{7}$ (ZMUM), $1 \sigma^{7}$ (NMNHS), China, Sichuan Prov, Lixian County, SW of Tonghua, $2810 \mathrm{~m}$ a.s. 1 , $31^{\circ} 30^{\prime} 00^{\prime \prime} \mathrm{N}$, $103^{\circ} 20^{\prime} 06^{\prime \prime} \mathrm{E}$, lower parts of a broadleaved forest, 10.07.2012, leg. I.A. Belousov \& G.E. Davidian; $1 \sigma^{7}, 1$ (ZMUM), $1 \sigma^{7}$ (ZIN), Sichuan Prov., Jiuzhaigou County, N of Dajisi, 3530-4000 m a.s.1., $33^{\circ} 22^{\prime} 43^{\prime \prime} \mathrm{N}-33^{\circ} 23^{\prime} 20^{\prime \prime} \mathrm{N}, 103^{\circ} 48^{\prime} 26^{\prime \prime} \mathrm{E}-103^{\circ} 49^{\prime} 01^{\prime \prime} \mathrm{E}$, broadleaved forest, 21.06.2012, leg. I.A. Belousov, G.E. Davidian,

I.I. Kabak \& A.E. Korolev; 3 フ $\sigma^{7}, 1$ (ZMUM), 1 ○ (ZIN), Sichuan Prov., Maoxian County, SE of Nanxinzhen, $4170 \mathrm{~m}$ a.s.1., $31^{\circ} 34^{\prime} 01^{\prime \prime} \mathrm{N}, 103^{\circ} 49^{\prime} 16^{\prime \prime} \mathrm{E}$, subalpine meadows, 2.07.2012, leg. I.A. Belousov \& G.E. Davidian; $3 \sigma^{7} \sigma^{7}$ (ZMUM), Sichuan Prov., Baoxing-Xiaojin Pass, $4085 \mathrm{~m}$ a.s.1., $30^{\circ} 52^{\prime} 20^{\prime \prime} \mathrm{N}, 102^{\circ} 41^{\prime} 01^{\prime \prime} \mathrm{E}$, subalpine meadows, 15.06.2012, leg. I.I. Kabak \& A.E. Korolev; 1 $\sigma^{7}$ (ZMUM), Sichuan Prov., Juizhaigou County, N of Dajisi, 4205 

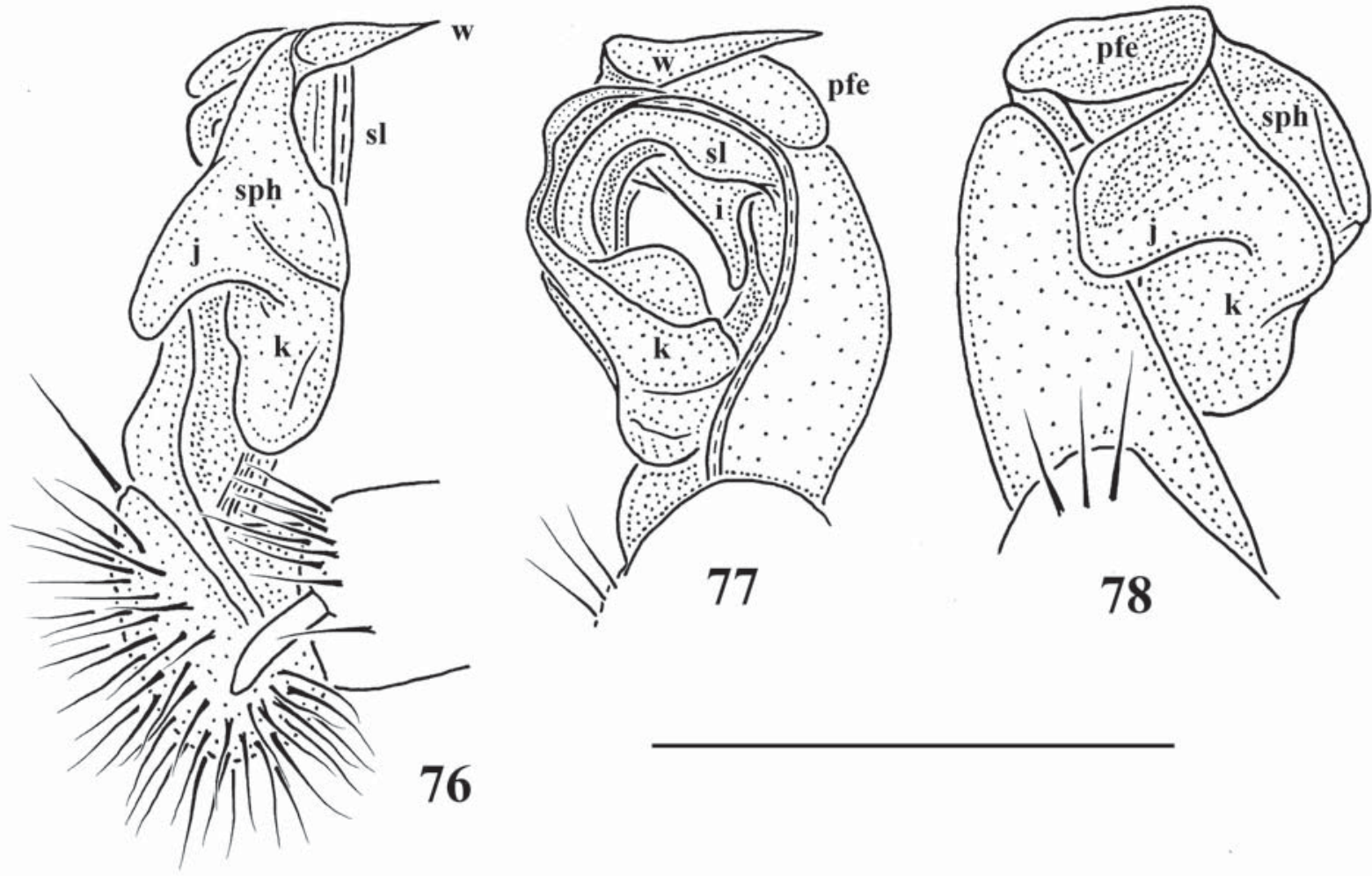

Figs 76-78. Right gonopod of Sigipinius grahami Hoffman, 1961, O from SE of Shangxinzhen: mesal, dorsal and ventral, views, respectively (spine $\mathbf{w}$ likely an artifact). Scale bar: $1.0 \mathrm{~mm}$.

Рис. 76-78. Правый гонопод Sigipinius grahami Hoffman, 1961, О' из юго-восточнее Shangxinzhen: соответственно изнутри, сверху и снизу (шип w, скорее всего, артефакт). Масштаб 1,0 мм.

m a.s.1., $33^{\circ} 23^{\prime} 32^{\prime \prime} \mathrm{N}, 103^{\circ} 48^{\prime} 11^{\prime \prime} \mathrm{E}$, subalpine and alpine meadows, 23.06.2012, leg. I.A. Belousov \& G.E. Davidian; $20^{7} 0^{7}, 1$ क (ZMUM), same locality, $3300 \mathrm{~m}$ a.s.1., $33^{\circ} 22^{\prime} 22^{\prime \prime} \mathrm{N}, 103^{\circ} 47^{\prime} 28^{\prime \prime} \mathrm{E}$, 24.06.2012; $1 \mathrm{O}^{7}$ (ZMUM, same locality, $2600 \mathrm{~m}$ a.s.1., $33^{\circ} 20^{\prime} 32^{\prime \prime} \mathrm{N}$ $103^{\circ} 48^{\prime} 46^{\prime \prime} \mathrm{E}$, lower parts of a broadleaved forest, 20.06.2012; 3 $\mathrm{O}^{7} \mathrm{O}^{7}$ (ZMUM), Sichuan Prov., W of Juizhaigou, $2900 \mathrm{~m}$ a.s.1. broadleaved forest, $33^{\circ} 21^{\prime} 19^{\prime \prime} \mathrm{N}, 103^{\circ} 48^{\prime} 05^{\prime \prime} \mathrm{E}, 21.06 .2012$, all leg. I.A. Belousov, G.E. Davidian, I.I. Kabak \& A.E. Korolev.

DIAGNOSIS. Differs from congeners except $S$. kabaki sp.n. largely by the deeply bifurcate solenophore (branches $\mathbf{j}$ and $\mathbf{k}$ ), from $S$. kabaki sp.n. by the slightly different solenophore which shows a peculiar ridge (z) proximal to both branches $\mathbf{j}$ and $\mathbf{k}$, of which the latter is larger than the former, not vice versa. See also Key below.

REDESCRIPTION. Length ca 21-27 $\left(\bigcirc^{7}\right)$ or 23$31 \mathrm{~mm}(+)$, width of midbody pro- and metazona 1.8 2.7 and 2.4-3.1 $\left(\sigma^{7}\right), 2.6-3.2$ and 2.9-3.5 mm (+), respectively, versus ca $26 \mathrm{~mm}$ long and $2.5 \mathrm{~mm}$ wide indicated for $\sigma^{\top}$ holotype [Hoffman, 1961]. General coloration in alcohol usually blackish to dark brown, more rarely nearly uniformly red-brown, tips of antennae invariably whitish; pattern evident as an oblong, axial, yellowish spot on collum, a similar, but shorter, rounded spot in rear half of following metaterga 2-19, and a fully yellowish epiproct (Figs 65-67).

In width, head $<$ collum $=$ segment $3<2=4<5$ 16; thereafter body gradually tapering towards telson. Clypeolabral region densely setose, vertigial one with only a few setae. Antennae rather short, clavate, reach- ing behind midway of body segment $2\left(\sigma^{7}\right)$ or behind collum (+) when stretched dorsally; antennomeres 2-6 subequal in length, but $6^{\text {th }}$ slightly higher. Paraterga very poorly developed (Figs 65-68), especially so in 9 , set rather high, mostly lying at about $1 / 3$ of midbody height, never produced behind rear margin; paraterga starting from collum, broadly rounded lobes; $2^{\text {nd }}$ particularly low, a distinct, large, broadly rounded lobe; following poreless paraterga considerably thinner than laterally very flat pore-bearing ones, $19^{\text {th }}$ missing; calluses very weak, devoid of lateral indentations, usually delimited by a modest sulcus only dorsally, sometimes slightly outlined also below by a longitudinal wrinkle, usually also followed by a few, similar, longitudinal wrinkles on sides. Ozopores lateral, mostly an inconspicuous round or slightly ovoid pit lying at about $1 / 4$ of metazonite's length in front of caudal margin (Figs $65,68)$. Body surface generally smooth and shining, postsulcus halves of metaterga at most faintly rugulose, but sides below paraterga rugulose to rugose and microgranulate. Axial line missing to barely traceable in places on metaterga alone. Transverse metatergal sulcus very thin and incomplete, far from reaching bases of paraterga, present in segments 5-16(17), absent from $18^{\text {th }}$ and $19^{\text {th }}$. Tergal setae mostly abraded, at least two transverse rows on collum (about $3+3$ near front margin, about $2+2$ near middle), pattern on following metaterga sometimes traceable as $2+2$ or $3+3$ short 
setae or weak insertion points arranged in a single row in front of sulcus. Stricture dividing pro- and metazona rather deep, finely and densely striolate down to below paraterga (Fig. 68). Pleurosternal carinae very evident, especially so in $\sigma^{7}$, arcuate ridges, increasingly strongly developed until segment 7 (Fig. 65), thereafter abruptly reduced, but traceable until segment 15 , small bulges on $16^{\text {th }}$, onward missing; a small, rounded, subcaudal tooth present only in segments $2-7$, thereafter wanting. Epiproct (Figs 65, 67, 69) rather long, flattened dorsoventrally, tip subtruncate, subapical papillae small. Hypoproct (Fig. 70) semi-circular, caudal 1+1 setae clearly separated and not borne on knobs.

Sternites without modifications, densely setose; cross-impressions rather modest, axial impression especially weak; a pair of paramedian, rather high, apically densely setose, medially from subcontiguous to separated lobes between coxae 4 (Fig. 71). Legs medium-sized, in $\sigma^{7}$ somewhat incrassate compared to $q$ (Figs 65,68$)$, ca $1.2-1.3$ times $\left(\sigma^{7}\right)$ or about as long as midbody height ( $(+)$; prefemora without evident dorsal bulges; in $\sigma^{7}$, distal parts of tibae and all tarsi until two last leg-pairs with ventral brushes; adenostyles absent.

Gonopods (Figs 72-78) complex; coxite rather short, subcylindrical, densely setose ventro-apically; prefemoral part as usual, densely setose, rather short, about 1/ 3 the length of acropodite and only slightly shorter than femorite, the latter simple, devoid of outgrowths, with a mesal impression and a seminal groove at bottom; postfemoral region (pfe) short, subcylindrical, simple, demarcated by a distinct sulcus each from femorite basally and from solenophore (sph) apically; sph strongly curved, directed mesad, very large, unifoliate, represented solely by a lamina lateralis, deeply bifurcate in distal half (branches $\mathbf{k} \& \mathbf{j}$, both flattened and slightly variable in shape, but $\mathbf{j}$ always clearly smaller than $\mathbf{k}$ ), not sheathing a long, flagelliform solenomere (sl) which is neatly attached to mesal face of sph. The latter at base always supplied with an evident, rather long, subdigitiform, ventral process (i) and sometimes also with a nearby lobule corresponding to $\mathbf{u}$; an apical membranous spine $\mathbf{w}$ obviously being an artifact absent from the opposite gonopod and all other $\sigma^{7}$ specimens.

REMARKS. The genus Sigipinius Hoffman, 1961 has hitherto been monobasic, with S. grahami Hoffman, 1961, from Si Gi Pin, Omei Shan Mountain $\left(29^{\circ} 35^{\prime} \mathrm{N}, 103^{\circ} 11^{\prime} \mathrm{E}, 6000\right.$ feet a.s.1.), Sichuan, China, being the type species [Hoffman, 1961]. The genus has since been assigned to and remained placed in the mostly East Asian tribe Sulciferini [Jeekel, 1968, 1988; Hoffman, 1980].

The original description is fairly complete, and the gonopod drawings very clear [Hoffman, 1961], to be certain about the identification of the new samples which may well be considered as near-topotypes. The above detailed redescription and new illustrations are provided not only to outline the rather pronounced variation range of $S$. grahami in size, coloration, gono- pod structure etc., but also for comparative purposes vis-à-vis four new congeners described below.

Golovatch [2011], when proposing the monotypic genus Chinomorpha Golovatch, 2011, with C. montana Golovatch, 2011, from Yunnan, China, as the type species, paid due attention to its gonopod conformation, in particular, the presence of a clear-cut postfemoral region, the large, unifoliate solenophore (= lamina lateralis), and the long, flagelliform solenomere neatly attached to the mesal side of, but virtually not sheathed by, the solenophore. The latter two features are characteristic of the largely tropical Asian tribe Sundaninini, wherein Chinomorpha was immediately and rightly placed. However, the previously overlooked resemblance of $C$. montana to $S$. grahami, both in somatic (e.g. the same colour pattern, very poorly developed paraterga, strongly developed pleurosternal carinae, $\sigma^{7}$ tarsal brushes present etc.) and gonopod traits, is so clear that there can be no doubt that these two species are congeneric. Therefore, Sigipinius Hoffman, 1961 must be considered as a new senior subjective synonym of Chinomorpha Golovatch, 2011, syn.n., also to be transferred to Sundaninini, comb.n. Furthermore, in addition to $S$. montanus, comb.n. ex Chinomorpha, and $S$. grahami, four new species have been revealed, all in China as well, thus allowing for the diagnosis of Sigipinius to be refined, and all of its six constituent species keyed (see below).

\section{Sigipinius campanuliformis sp.n. Figs 79-90.}

HOLOTYPE $\sigma^{7}$ (ZMUM), China, Yunnan Prov., W of Lake Lugu, $\mathrm{N}$ of Dajisi, $3910 \mathrm{~m}$ a.s.1., $27^{\circ} 41^{\prime} 57^{\prime \prime} \mathrm{N}, 100^{\circ} 34^{\prime} 06^{\prime \prime} \mathrm{E}$, upper timberline of a broadleaved forest with dry forest edges and low bushes, 29.05.2012, leg. I.A. Belousov, G.E. Davidian, I.I. Kabak \& A.E. Korolev.

NAME. To emphasize the bell-shaped solenophore.

DIAGNOSIS. Differs from congeners mainly by the peculiar, bell-shaped solenophore. See also Key below.

DESCRIPTION. Length ca $25 \mathrm{~mm}$, width of midbody pro- and metazona 2.5 and $3.0 \mathrm{~mm}$, respectively. General coloration in alcohol uniformly blackish, much like in $S$. grahami, but pattern a little more vague, including dark brown head and legs, smaller and less clear-cut dorsal spots on collum and following metaterga, and very small light spots in front of ozopores (Figs $79 \& 83)$.

All other characters like in S. grahami, except as follows.

In width, segments $3 \& 4<$ head $=$ collum $<2<5-$ 16 (Figs $79 \& 80$ ). Transverse sulcus on metaterga vestigial, only in places traceable as a very weak impression (Figs 79, 81-84). Epiproct tip clearly concave (Fig. 85).

Gonopods (Figs 88-90) with a bell-shaped solenophore (sph) barely supporting a usual, flagelliform solenomere (sl) on mesal side alone; process $\mathbf{i}$ at base of $\mathbf{s p h}$ vestigial, but postfemoral region (pfe) a little enlarged. 

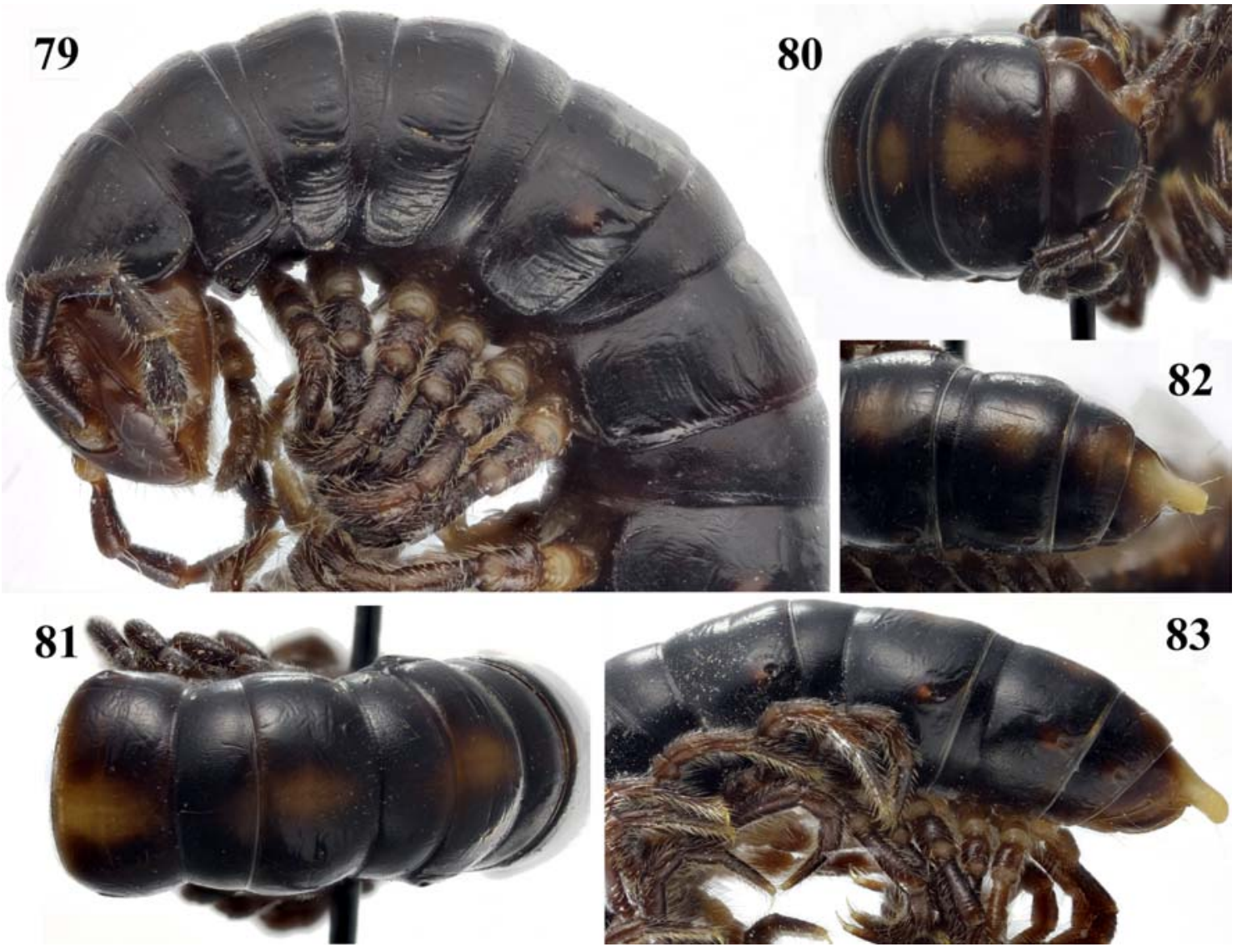

Figs 79-83. Sigipinius campanuliformis sp.n., holotype: $79 \& 80$ - anterior body part, lateral and dorsal views, respectively; 81 midbody segments, dorsal; $82 \& 83$ - caudal body parts, subdorsal and lateral views, respectively. Photos taken by K. Makarov, not to scale.

Рис. 76-83. Sigipinius campanuliformis sp.n., голотип: 79 и 80 - передняя часть тела, соответственно сбоку и сверху; 81 среднетуловищные сегменты, сверху; 82 и 83 - задняя часть тела, соответственно почти свенрху и сбоку. Фото сделаны К. Макаровым, без масштаба.

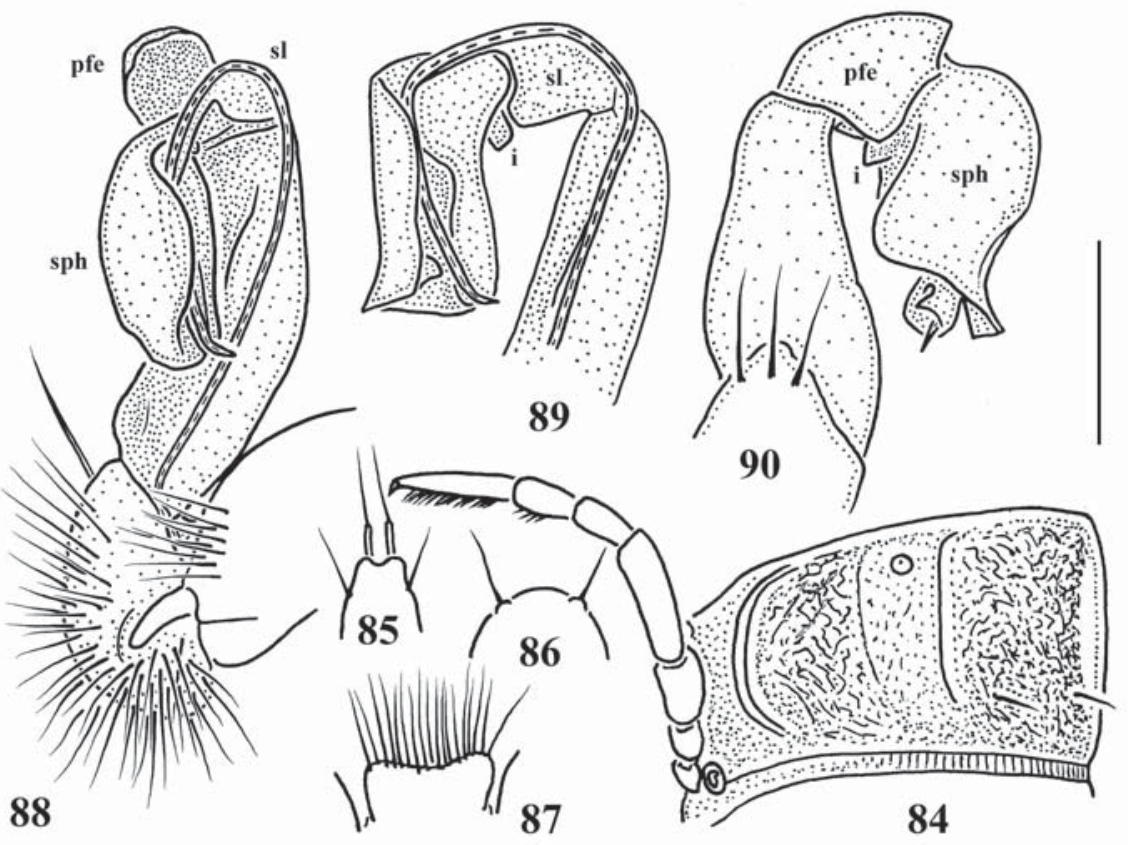

Figs 84-90. Sigipinius campanuliformis sp.n., holotype: 84 body segment 10, lateral; 85 epiproct tip, dorsal; 86 - hypoproct, ventral; 87 - sternal lobe between coxae 4, caudoventral view; 88-90 - right gonopod, mesal, subdorsal and ventral views, respectively. Scale bar: 1.35 (84$87)$ and $0.5 \mathrm{~mm}(88-90)$.

Рис. 84-90. Sigipinius campanuliformis sp.n., голотип: 84 - сегмент 10, сбоку; $85-$ эпипрокт, сверху; 86 - гипопрокт, снизу; 87 - стернальная пластина между тазиками 4 , вид одновременно снизу и сзади; 8890 - правый гонопод, соответственно изнутри, почти сверху и снизу. Масштаб 1,35 (84-87) и 0,5 мм (88-90). 


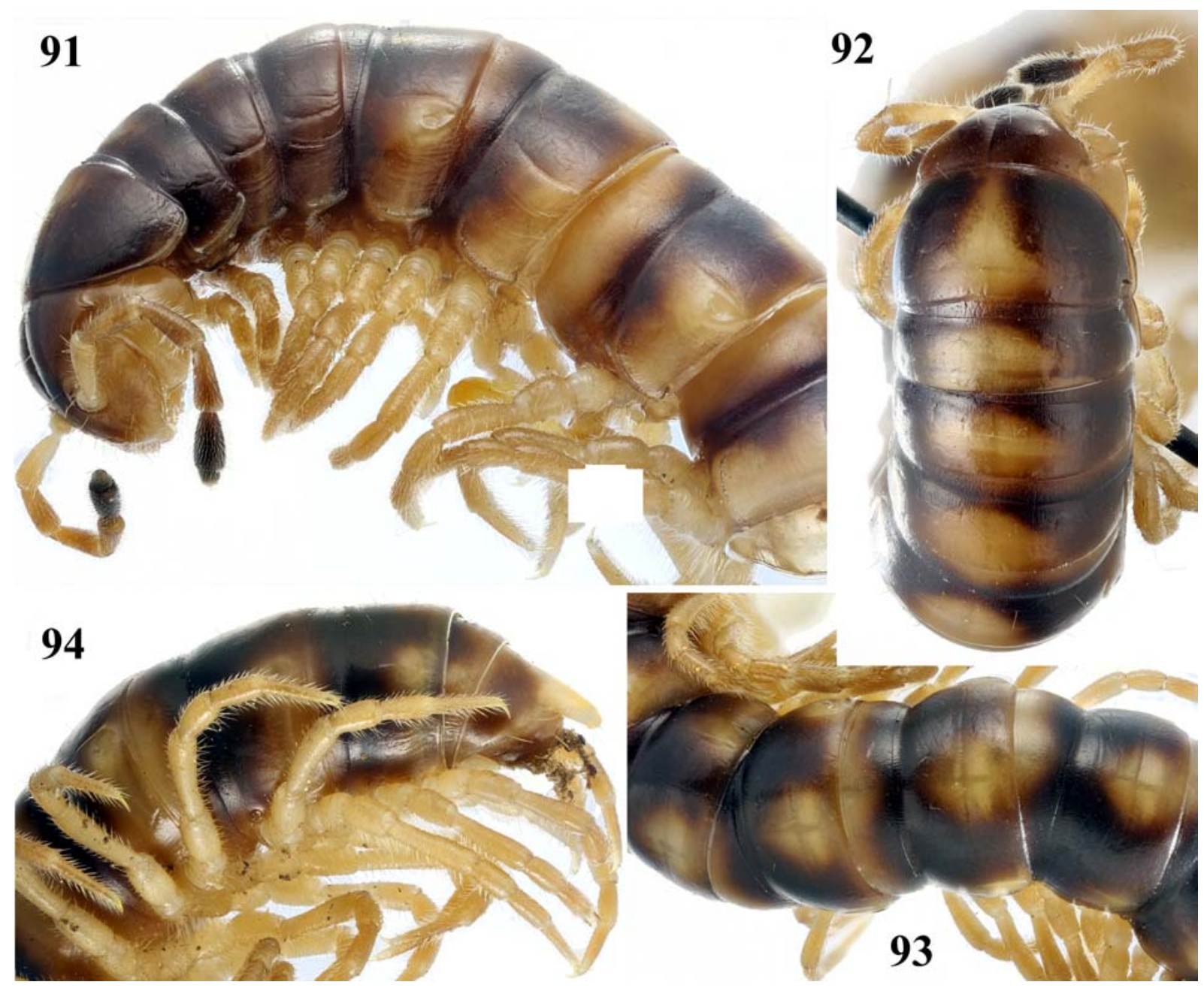

Figs 91-94. Sigipinius complex sp.n., $\sigma^{7}$ paratype from S of Muli, 3975-4120 m a.s.1.: 91 \& 92 - anterior body part, lateral and dorsal views, respectively; 93 - midbody segments, dorsal; 94 - caudal body part, lateral. Photos taken by K. Makarov, not to scale.

Рис. 91-94. Sigipinius complex sp.n., паратип О7 из южнее Muli, 3975-4120 м н.у.м.: 91 и 92 - передняя часть тела, соответственно сбоку и сверху; 93 - среднетуловищные сегменты, сверху; 94 - задняя часть тела, соответственно почти сверху и сбоку. Фото сделаны К. Макаровым, без масштаба.

\section{Sigipinius complex sp.n.}

Figs 91-104.

HOLOTYPE $0^{7}$ (ZMUM), China, Sichuan Prov., S of Muli, $3780 \mathrm{~m}$ a.s.1., $27^{\circ} 45^{\prime} 24^{\prime \prime} \mathrm{N}, 101^{\circ} 15^{\prime} 59^{\prime \prime} \mathrm{E}$, upper timberline of a broadleaved forest and subalpine meadows, 21.05.2012, leg. I.A. Belousov, G.E. Davidian, I.I. Kabak \& A.E. Korolev.

PARATYPES: $1 \sigma^{\gamma}, 1$ (ZMUM), same data, together with holotype; $3 \bigcirc^{7} \Im^{7}, 3$ 우, 19 fragment (ZMUM), $1 \Im^{7}, 2$ 우 (ZIN), Sichuan Prov., S of Muli, 3975-4120 m a.s.1., 27 $43^{\prime} 16^{\prime \prime}-27^{\circ}$ $43^{\prime} 03^{\prime \prime} \mathrm{N}, 101^{\circ} 15^{\prime} 13^{\prime \prime}-101^{\circ} 15^{\prime} 05^{\prime \prime} \mathrm{E}$, subalpine meadows, 22.05. 2012, leg. I.A. Belousov, G.E. Davidian, I.I. Kabak \& A.E. Korolev.

NAME. To emphasize the particularly complex solenophore.

DIAGNOSIS. Differs from congeners mainly by the peculiar colour pattern and solenophore structure. See also Key below.

DESCRIPTION. Length ca 26-27 ( $0^{\top}$, , $)$, width of midbody pro- and metazona 2.6 and $3.0\left(\mathrm{O}^{\top}\right)$ or 3.1 and $3.4 \mathrm{~mm}(+)$, respectively. General coloration in alcohol brown, pattern much like in S. grahami or S. campanuliformis sp.n., but considerably lighter and clearer, also somewhat cingulate due to invariably infuscate regions of stricture between pro- and metazona: antennae increasingly infuscate towards dark brown antennomeres 6 and 7 (Figs $91 \& 92$ ), lighter areas much larger, covering not only central parts of collum and following metaterga, but also front parts of prozona, ozopore regions and even lateral parts of metazona below paraterga (Figs 91-94).

All other characters like in S. grahami, except as follows.

In width, head $<$ collum $=$ segments $3 \& 4<2<5$ 15(16) (Fig. 92). Calluses on paraterga mostly delimited by a sulcus only dorsally (Figs 91, 93, 95), especially poorly developed in + . Transverse sulcus on metaterga vestigial, only in places traceable as a very weak impression (Figs 91-95). Axial line very weak, but traceable at least on metaterga (Figs $92 \& 93$ ). 

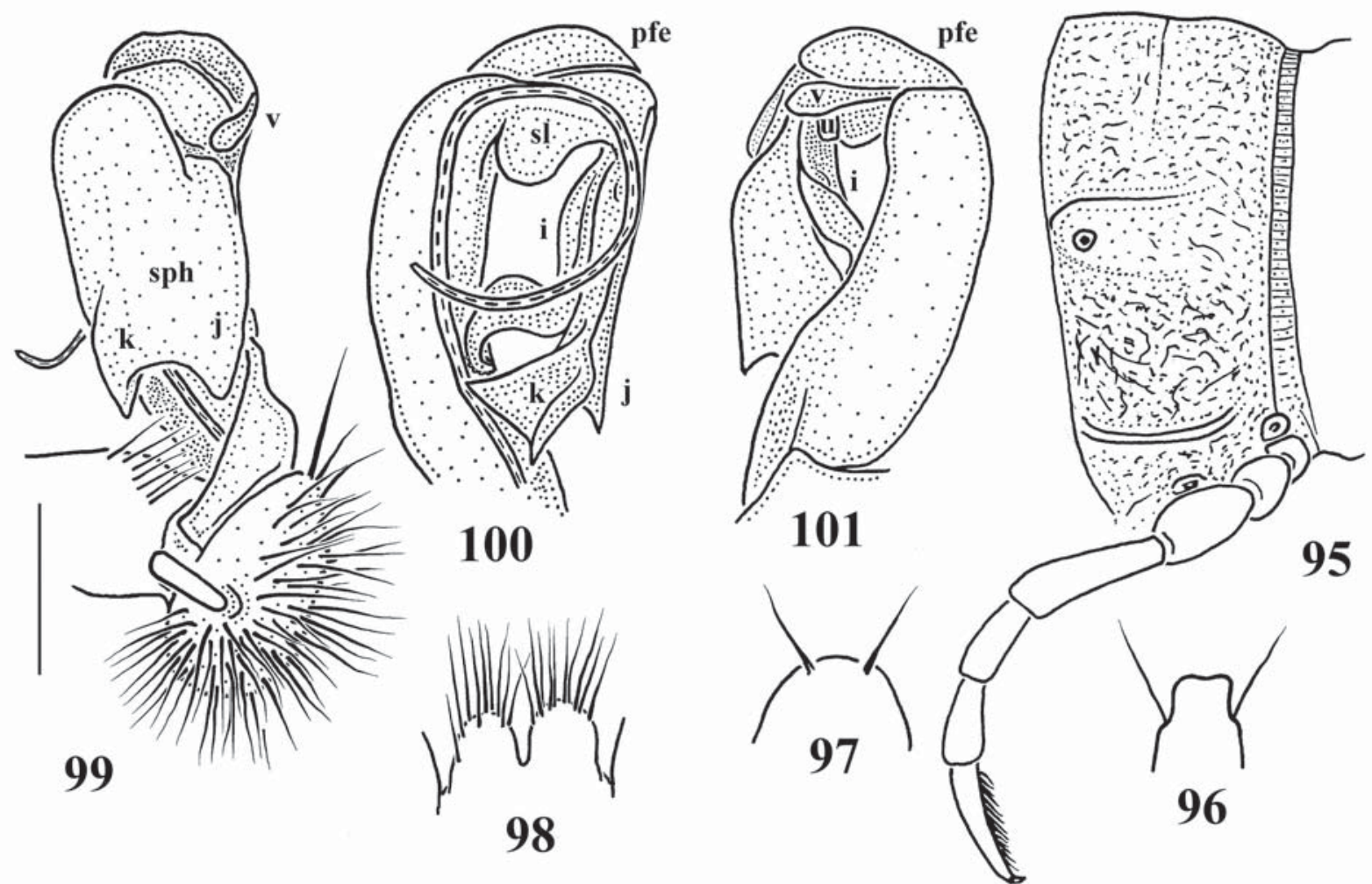

Figs 95-101. Sigipinius complex sp.n., O paratype from S of Muli, 3975-4120 m a.s.1.: 95 — body segment 10, lateral; 96 - epiproct tip, dorsal; 97 - hypoproct, ventral; 98 - sternal lobe between coxae 4, caudoventral view; 99-101 - left gonopod, mesal, dorsal and ventral views, respectively. Scale bar: 2.0 (95-98) and $0.5 \mathrm{~mm}(99-101)$.

Рис. 95-101. Sigipinius complex sp.n., паратип о7 из южнее Muli, 3975-4120 м н.у.м.: 95 - сегмент 10, сбоку; 96 - эпипрокт, сверху; 97 - гипопрокт, снизу; 98 - стернальная пластина между тазиками 4, одновременно снизу и сзади; 99-101 - левый гонопод, соответственно изнутри, сверху и снизу. Масштаб 2,0 (95-98) и 0,5 мм (99-101).

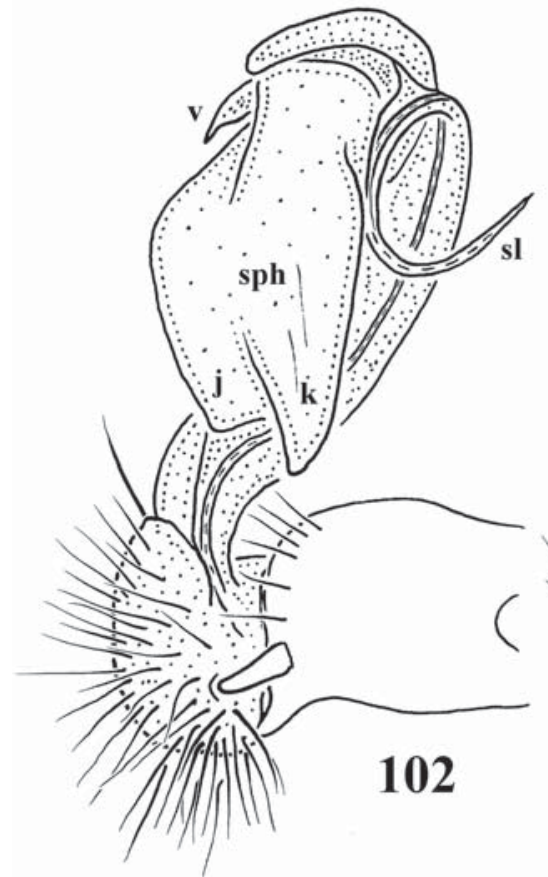

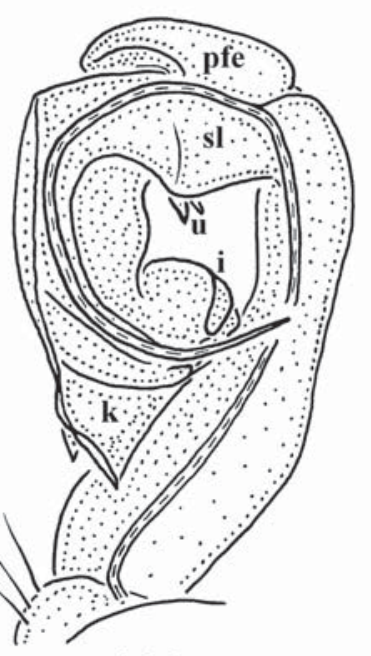

103

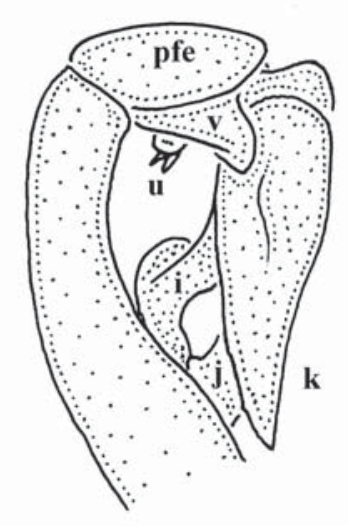

104

Figs 102-104. Right gonopod of Sigipinius complex sp.n., $\sigma^{7}$ paratype from S of Muli, 3975-4120 m a.s.1.: mesal, dorsal and ventral views, respectively. Scale bar: $2.0 \mathrm{~mm}$.

Рис. 102-104. Правый гонопод Sigipinius complex sp.n., паратип О7 из южнее Muli, 3975-4120 м н.у.м.: соответственно изнутри, сверху и снизу. Масштаб 2,0 мм. 

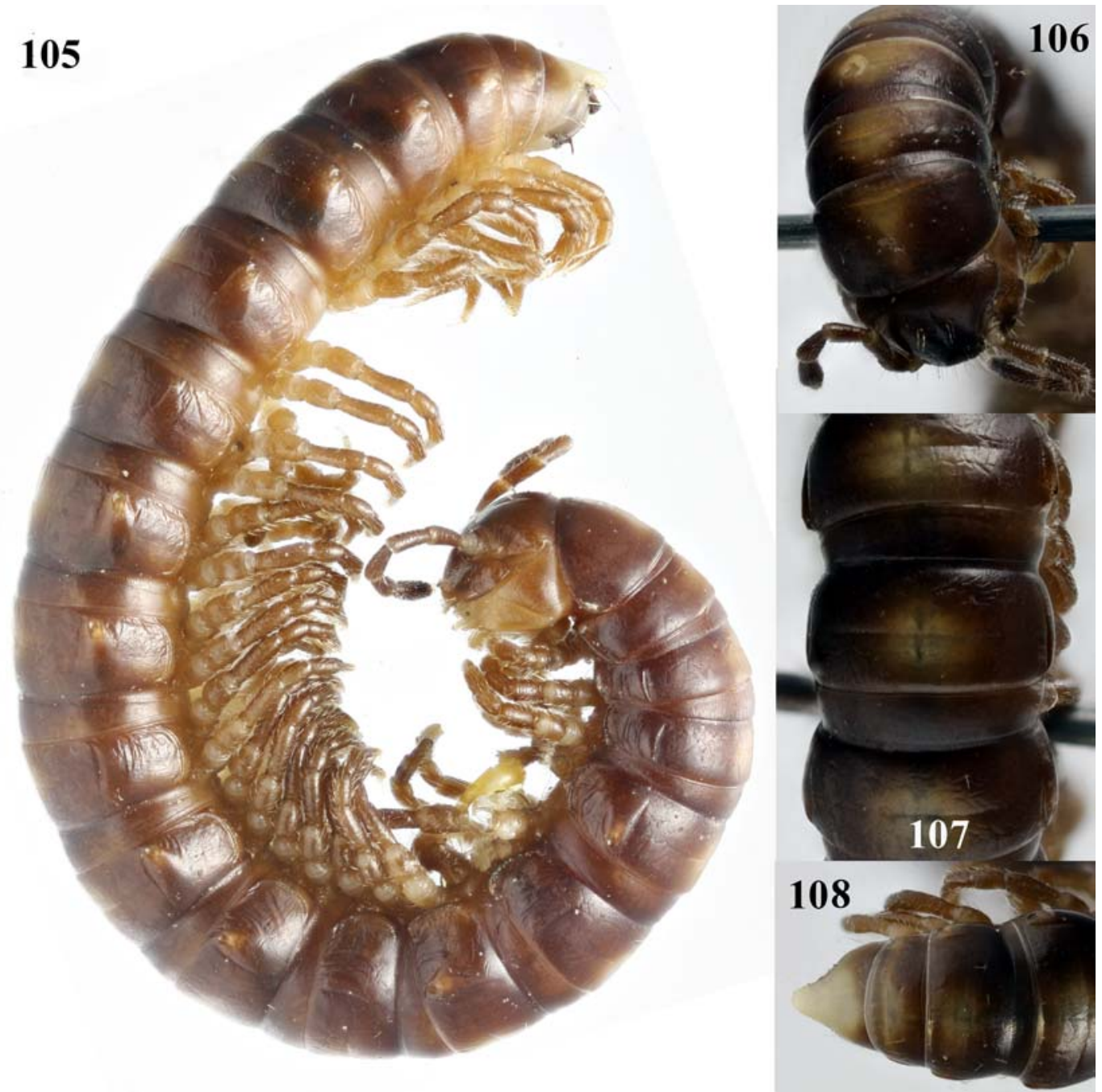

Figs 105-108. Sigipinius simplex sp.n., O paratype from Jiulong County: 105 — habitus, lateral; 106-108 — anterior body part, midbody segments and caudal body part, respectively, dorsal. Photos taken by K. Makarov, not to scale.

Рис. 105-108. Sigipinius simplex sp.n., паратип О7 из общины Jiulong: 105 - общий вид, сбоку; 106-108 - соответственно передняя часть тела, среднетуловищные сегменты и задняя часть тела, сбоку. Фото сделаны К. Макаровым, без масштаба.

Pleurosternal carinae much more poorly developed in o compared to $\sigma^{7}$ (Figs $\left.91 \& 95\right)$, also traceable until segment 17. Sternal lamina between $\sigma^{7}$ coxae 4 divided into two distinct, medially separated, densely setose lobes (Fig. 98). Legs in 9 considerably slenderer and shorter than in $\sigma^{7}$ (also about 1.0 versus $1.2-1.3$ as long as midbody height), but distotibial and tarsal brushes gradually thinning out towards legs of $\sigma^{7}$ segment 12 or 13 .

Gonopods (Figs 99-104) with a particularly complex, only faintly bifid (branches $\mathbf{j}$ and $\mathbf{k}$ ) solenophore (sph) barely supporting a usual, flagelliform solenomere (sl) on mesal side alone; base of sph supplied both with an especially large, sigmoid process $\mathbf{i}$ and a vestigial, sometimes bispinulate outgrowth (u) on ventral side, while postfemoral region (pfe) not too large, but supplied with a rather small, longitudinally elongate, ventral lobe (v).

\section{Sigipinius simplex sp.n.}

Figs 105-116.

HOLOTYPE OT (ZMUM), China, Sichuan Prov., Jiulong County, SW of Wulaxixiang, $4050 \mathrm{~m}$ a.s.1., $28^{\circ} 34^{\prime} 40^{\prime \prime} \mathrm{N}, 101^{\circ} 35^{\prime} 33^{\prime \prime} \mathrm{E}$, alpine meadows, 13.06.2012, leg. I.A. Belousov \& G.E. Davidian.

PARATYPES: $10^{7}$ (ZMUM), same data, together with holotype; $1 \sigma^{7}, 1$ (ZMUM). Sichuan Prov., Muli County, SW of Wulaxixiang, $4195 \mathrm{~m}$ a.s.1., $28^{\circ} 32^{\prime} 49^{\prime \prime} \mathrm{N}, 101^{\circ} 34^{\prime} 13^{\prime \prime} \mathrm{E}$, alpine meadows, 15.06.2012, leg. I.A. Belousov \& G.E. Davidian.

NAME. To emphasize the particularly simple gonopod structure.

DIAGNOSIS. Differs from congeners mainly by the particularly simple postfemoral region and solenophore. See also Key below.

DESCRIPTION. Length ca 20-22 ( $\left.\sigma^{7},+\right)$, width of midbody pro- and metazona $1.7-1.8$ and $2.0-2.1\left(\bigcirc^{7}\right)$ or 2.0 and $2.2 \mathrm{~mm}\left({ }_{+}\right)$, respectively. General coloration in alcohol from rather uniformly brown to blackish, pattern 


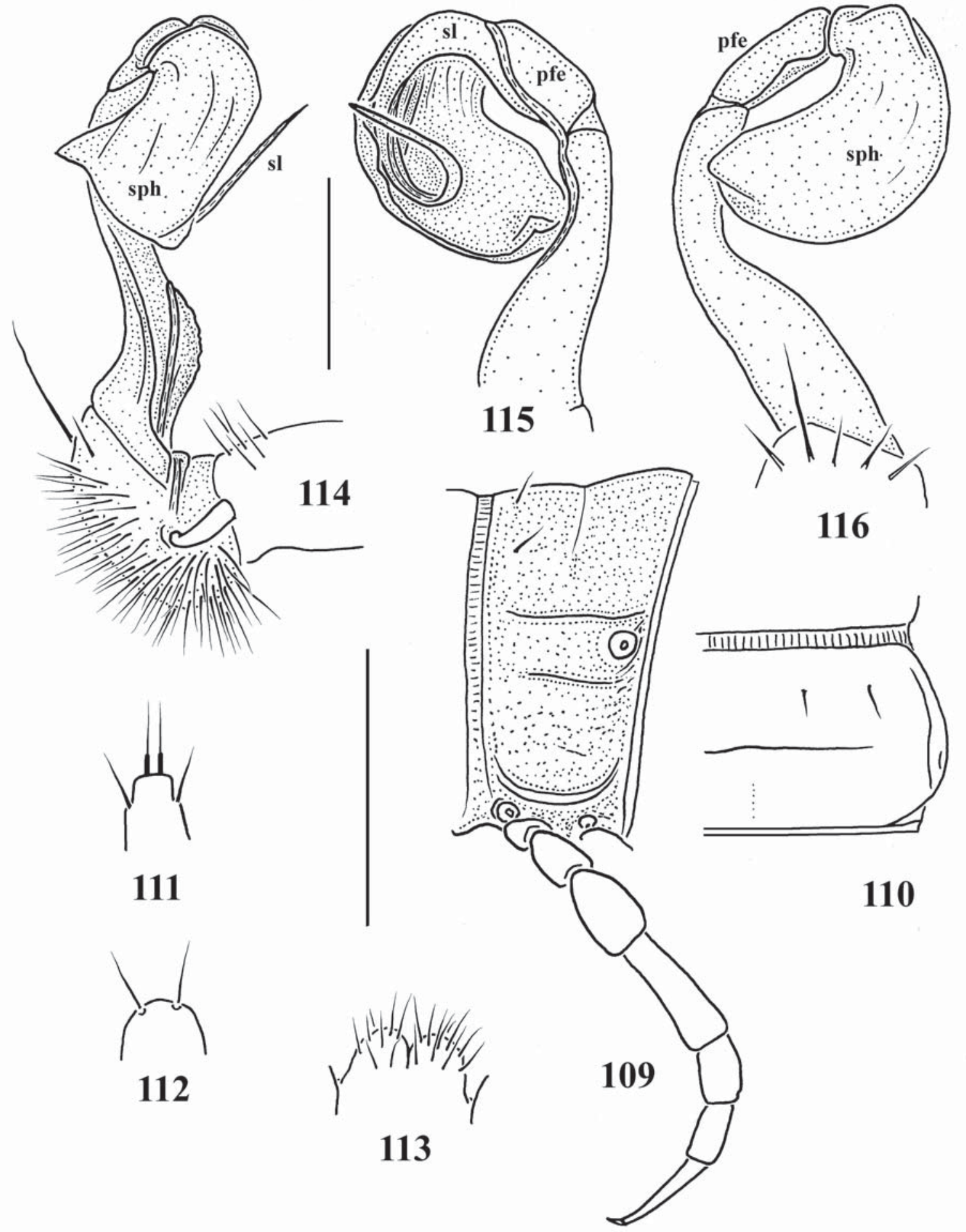

Figs 109-116. Sigipinius simplex sp.n., $0^{7}$ paratype from Jiulong County: $109 \& 110$ — body segment 10, lateral and dorsal views, respectively (tarsal and distotibial brushes not shown); 111 — epiproct, dorsal; 112 — hypoproct, ventral; 113 — sternal lobe between coxae 4, caudoventral view; 114-116 — right gonopod, mesal, dorsal and ventral views, respectively. Scale bars: 1.0 (109-113) and 0.5 $\mathrm{mm}(114-116)$.

Рис. 109-116. Sigipinius simplex sp.n., паратип О7 из общины Jiulong: 109 и 110 - сегмент 10, соответственно сбоку и сверху (тарсальные и дистотибиальные щетки не показаны); 111 - эпипрокт, сверху; 112 - гипопрокт, снизу; 113 - стернальная пластина между тазиками 4, одновременно снизу и сзади; 114-116 - правый гонопод, соответственно изнутри, сверху и снизу. Масштаб 1,0 (109-113) и 0,5 мм (114-116). 


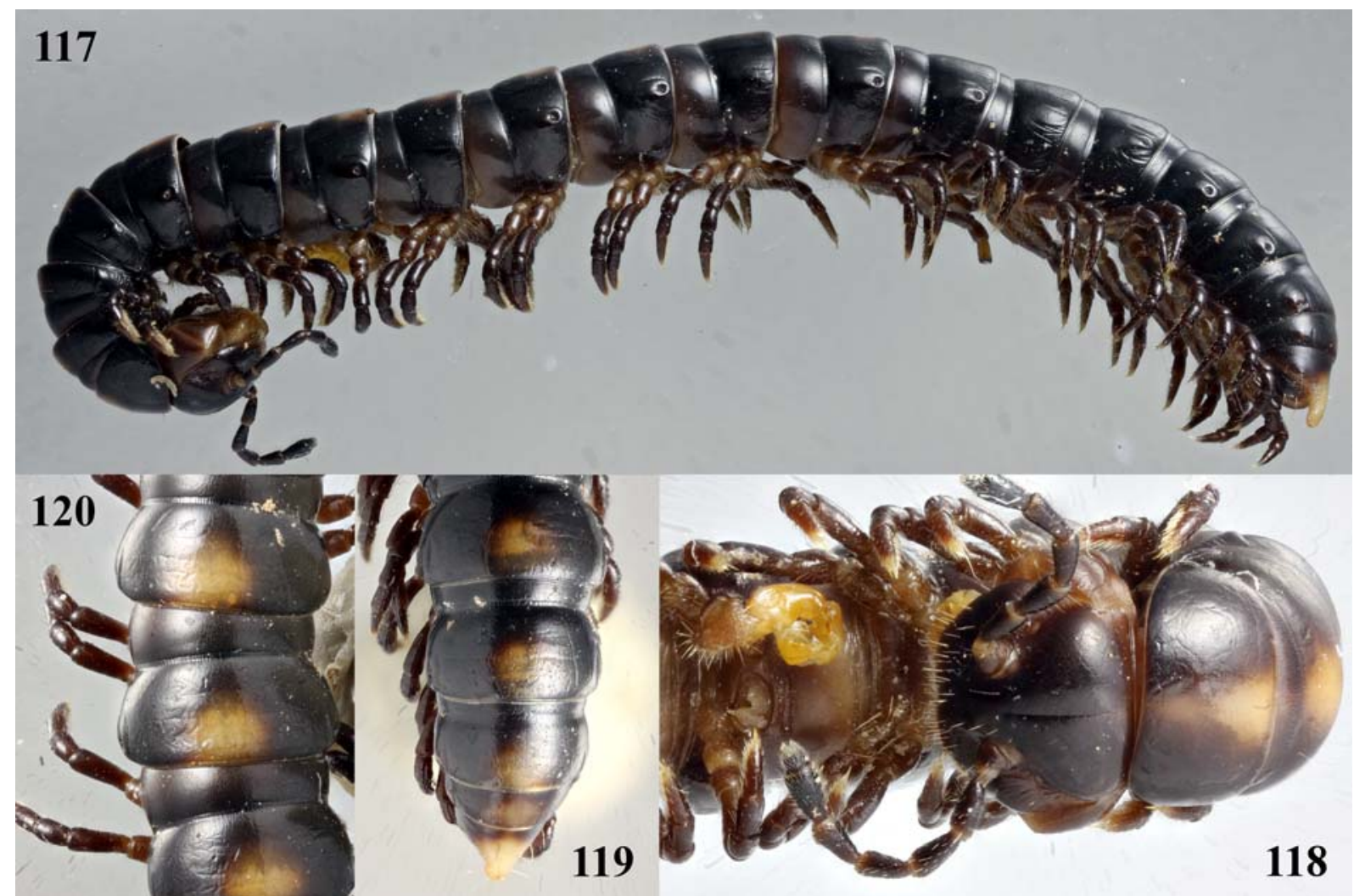

Figs 117-120. Sigipinius kabaki sp.n., holotype: 117 — habitus, lateral; 118 — anterior body part, front and ventral; 119 — midbody segments, dorsal; 94 - caudal body part, dorsal. Photos taken by K. Makarov, not to scale.

Рис. 117-120. Sigipinius kabaki sp.n., голотип: 117 - общий вид, сбоку; 118 - передняя часть тела, спереди и снизу; 119 среднетуловищные сегменты, сверху; 94 - задняя часть тела, сверху. Фото сделаны К. Макаровым, без масштаба.

much like in S. grahami or S. campanuliformis sp.n., but more vague, also somewhat cingulate due to infuscate stricture regions between pro- and metazona, light dorsal spots always visible (Figs 106-108).

All other characters like in $S$. grahami, except as follows.

In width, segments $3<$ head $=$ collum $<2=4<5$ 15(16) (Fig. 106). Calluses on paraterga mostly delimited by a sulcus both dorsally and ventrally, especially so in $\sigma^{\prime}$ (Figs $105 \&$ 109), underdeveloped in 9 . Transverse sulcus on metaterga thin, mostly quite visible in segments 5-17 (Figs 105, 107-110). Axial line very weak, but visible at least on metaterga (Figs $107 \& 108$ ). Pleurosternal carinae more poorly developed in + compared to $\sigma^{7}$ (Figs $105 \& 109$ ), traceable until segment 16 , with a subcaudal rounded denticle only in $\sigma^{7} \mathrm{seg}$ ment 7. Sternal lamina between $\sigma^{7}$ coxae 4 divided into two medially contiguous, densely setose lobes (Fig. 113). Legs in 9 considerably slenderer and shorter than in $\sigma^{7}$ (also about 1.0 versus $1.2-1.3$ as long and midbody height), but distotibial and tarsal brushes gradually thinning out towards legs of $\sigma^{7}$ segment 14 or 15.

Gonopods (Figs 114-116) with postfemoral part (pfe) and solenophore (sph) particularly simple, both devoid of evident outgrowths, sph (= only a large, foliate lamina lateralis) supporting a usual, long, flagelliform solenomere (sl) on mesal side alone.

\section{Sigipinius kabaki sp.n.}

Figs 117-127.

HOLOTYPE $0^{7}$ (ZMUM), China, Xinjiang Uygur Prov., Koeksu Basin, $3330-3550 \mathrm{~m}$ a.s.1., $42^{\circ} 32^{\prime} 47^{\prime \prime}-42^{\circ} 32^{\prime} 51^{\prime \prime} \mathrm{N}, 82^{\circ} 05^{\prime} 56^{\prime \prime}-$ $82^{\circ} 05^{\prime} 36^{\prime \prime} \mathrm{E}$, alpine meadows, 11.07 .2012 , leg. I.I. Kabak.

PARATYPE: 1 (ZMUM), same data, together with holotype.

NAME. Honours Ilya Kabak, of St. Petersburg, the collector.

DIAGNOSIS. Differs from congeners mainly by the peculiar solenophore structure. See also Key below.

DESCRIPTION. Length ca 29 (holotype) or 26 $\mathrm{mm}$ (paratype), width of midbody pro- and metazona 2.5 and 2.9 (holotype) or 2.6 and $2.9 \mathrm{~mm}$ (paratype), respectively. General coloration in alcohol dark brown, only antennae in holotype blackish; pattern much like in S. grahami (Figs 117-120).

All other characters like in S. grahami, except as follows.

In width, head $=$ segments $5-15(16)>$ segment $4>$ segments $3 \& 4>$ collum (Fig. 118). Antennomeres $3-$ 6 subequal in length, but $2^{\text {nd }}$ slightly longer (= longest). Calluses on paraterga delimited by sulci both dorsally and ventrally (Figs $117 \& 121$ ), especially poorly developed in + , surface below paraterga clearly rugose-rugulose, in places also microgranulate. Transverse 

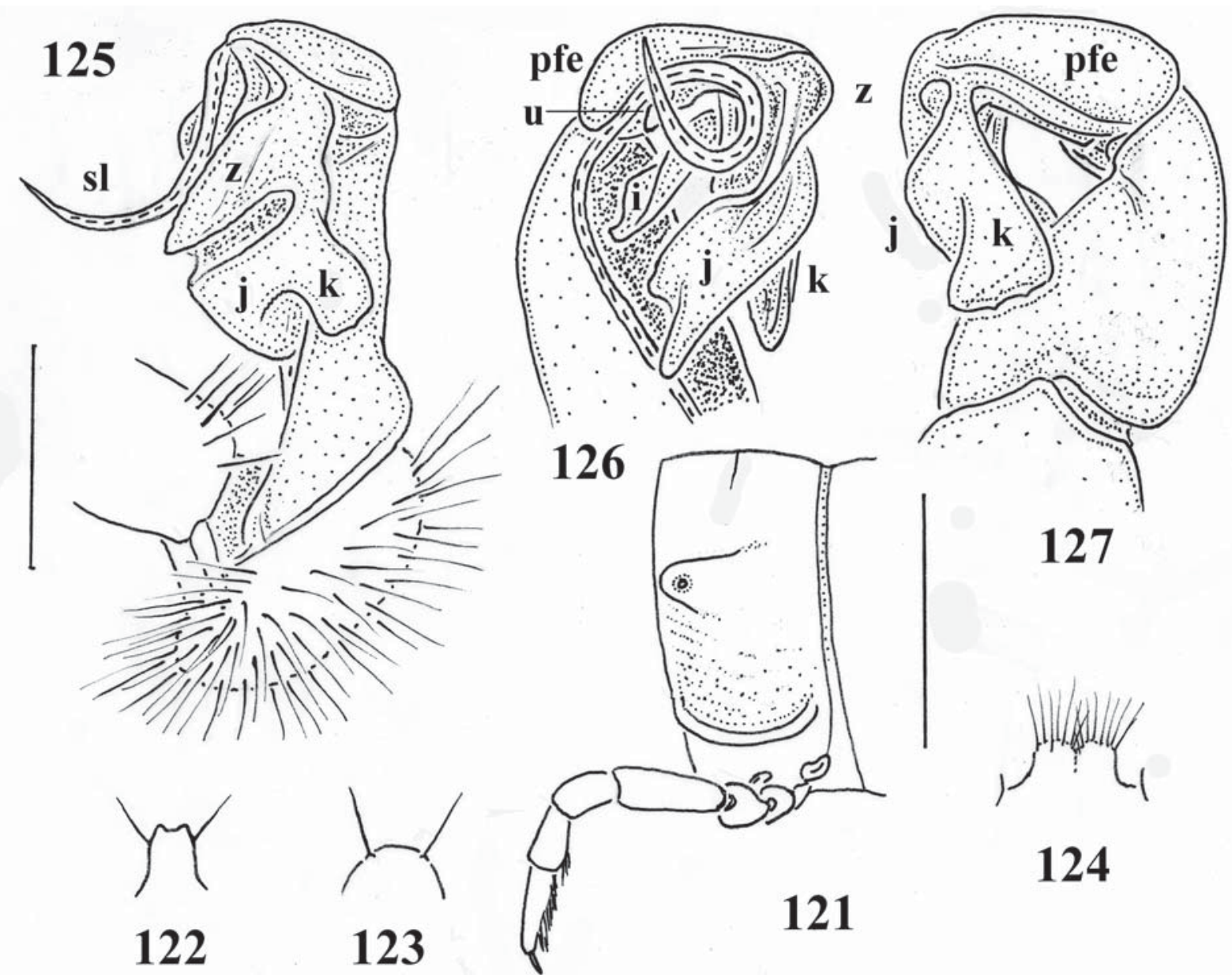

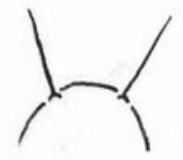

123

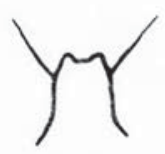

122

Figs 121-127. Sigipinius kabaki sp.n., holotype: 121 — body segment 10, lateral; 122 - epiproct, dorsal; 123 — hypoproct, ventral; 124 - sternal lobe between coxae 4, caudoventral view; 114-116 - left gonopod, mesal, dorsal and ventral views, respectively. Scale bars: $2.0(121-124)$ and $0.5 \mathrm{~mm}(125-127)$.

Рис. 121-127. Sigipinius simplex sp.n., голотип: 121 - сегмент 10, сбоку; 122 - эпипрокт, сверху; 123 - гипопрокт, снизу; 124 - стернальная пластина между тазиками 4, одновременно снизу и сзади; 125-127 - левый гонопод, соответственно изнутри, сверху и снизу. Масштаб 1,0 (121-124) и 0,5 мм (125-127).

sulcus on metaterga vestigial, only in places traceable until segment 18 as a very weak impression or line (Figs 119, 120 \& 121). Axial line very weak, but traceable in places on metaterga (Figs 118-120). Pleurosternal carinae much more poorly developed in $\rightarrow$ compared to $\sigma^{7}$, especially prominent and flap-shaped in $\sigma^{7}$ segments $2-8$, traceable as a very faint sulcus until segment $18\left(\mathrm{O}^{7}\right)$ or $17(+)$. Epiproct slightly concave at tip (Fig. 122). Hypoproct semi-circular, setigerous knobs small, inconspicuous (Fig. 123). Sternal lamina between $\sigma^{7}$ coxae 4 divided into two low, densely setose lobes contiguous medially at base (Fig. 124). Legs in $q$ considerably slenderer and shorter than in $\sigma^{7}$ (about 1.0 versus $1.2-1.3$ as long as midbody height), but distotibial brushes gradually thinning out towards legs of $\sigma^{7}$ segment 10, tarsal brushes until about legs of $\sigma^{7}$ segment 16 (Fig. 121).

Gonopods (Figs 125-127) with a complex, strongly bifid solenophore (sph), with branch $\mathbf{j}$ being much larger than a subquadrate branch $\mathbf{k}$, both lying distal to a conspicuous ridge (z); sph barely supporting a usual, flagelliform solenomere (sl) on mesal side alone; base of sph supplied both with a rather large, nearly straight, but somewhat helicoid process $\mathbf{i}$ and a vestigial outgrowth (u), while postfemoral region (pfe) quite prominent.

REMARKS. Considering the above new synonymy and four new species found, the genus Sigipinius can be rediagnosed as follows.

\section{Sigipinius Hoffman, 1961}

DIAGNOSIS. Medium-sized Sundaninini (ca 20$30 \mathrm{~mm}$ long) with a pattern of lighter spots against a dark backround at least mid-dorsally. Paraterga very poorly developed, especially so in + , sometimes delimited by a sulcus only dorsally, absent from segment 19 . Tergal setae short, on postcollum metaterga arranged in a single transverse front row of $2+2$ or $3+3$, pattern barely traceable when setae abraded. Pleurosternal carinae arcuate, strongly developed ridges, especially so until segment 7 , traceable until segment 17 , better de- 
veloped in $\sigma^{r}$ compared to + . A single transverse sternal lamina or a paramedian pair of setose lobes between $\sigma^{7}$ coxae 4 . Adenostyles absent. Ventral brushes present in most of $\sigma^{7}$ tarsi and, distally, tibiae.

Gonopod with a modest, subcylindrical, distally densely setose coxite only about half the length of telopodite. Prefemoral (= densely setose) part large, about $1 / 2-1 / 3$ the length of acropodite. Femorite considerably shorter and slenderer than a mesad strongly curved acropodite, devoid of any evidence of torsion, suberect to faintly curved also mesad, simple, lacking any outgrowths, mesally with an impression and a seminal groove at its bottom. Postfemoral part (pfe) evident, delimited by a sulcus each from femorite basally and from solenophore apically, usually simple, only sometimes with an additional small outgrowth (v) ventrally. Solenophore clearly foliate, represented only by a lamina lateralis, at base often with processes or outgrowths ( $\mathbf{u}$ and/or i), distally sometimes more or less deeply bifurcate (j and $\mathbf{k})$, loosely supporting a long, basically flagelliform solenomere (sl) only on mesal face, sl slightly expanded and ribbon-like near base, starting mesal to sulcus between femorite and pfe.

Type species: Sigipinius grahami Hoffman, 1961, by original designation.

DISTRIBUTION. All species of this genus seem to be high-montane (ranging from ca 2800 to $>4000 \mathrm{~m}$ a.s.l.) and confined to the south- and northwestern parts of China, currently being known only from Yunnan, Sichuan and Xinjiang Uygur provinces. Most likely, species of Sigipinius occur also at least in-between, i.e. in Qinghai Province.

The following key can be proposed for separating all six Sigipinius species known to date.

1(2) Gonopod postfemoral part and solenophore particularly simple, the latter leaf-shaped, devoid of strong outgrowths

2(1) Gonopod postfemoral part and, especially, solenophore more complex, the latter often with a strong process near base, sometimes also with a small nearby lobule ....... 5

3(4) Sternal lobe between $O^{7}$ coxae 4 entire, undivided mesally. A small spine present in basal $1 / 3$ of solenophore to support solenomere mesally. Yunnan ... S. montanus

4(3) A paramedian pair of sternal lobes between $O^{7}$ coxae 4 (Fig. 113). Solenophore devoid of a mesal spinule (Figs 114-116). Sichuan ................................. S. simplex sp.n.

5(6) Sternal lobe between $O^{7}$ coxae 4 entire, undivided mesally (Fig. 87). Solenophore strongly folded, bell-shaped, only a vestigial process $\mathbf{i}$ present at base of solenophore (Figs 88-90). Yunnan ........... S. campanuliformis sp.n.

6(5) A paramedian pair of sternal lobes between $O^{7}$ coxae 4. Solenophore different, more or less clearly divided distally into lobes $\mathbf{j}$ and $\mathbf{k}$, at base with a very evident process i and, often, also with a small lobule $\mathbf{u}$. Sichuan or Xinjiang .......................................................... 7

7(8) Coloration lighter, brown, pattern cingulate due to infuscate regions of strictures between pro- and metazona (Figs 91-94). Solenophore projections $\mathbf{j}$ and $\mathbf{k}$ less prominent, but process i remarkably large and strongly sigmoid (Figs 99-104)
8(7) Coloration darker, usually blackish to dark brown, pattern non-cingulate (Figs 65-67, 117-120). Solenophore deeply bifurcate, process i subdigitiform (Figs 72-78 \& 126)

9(10) Distal solenophore branch j clearly smaller than branch $\mathbf{k}$, an evident ridge proximal to both absent (Figs 7278). Sichuan ............................................... S. grahami 10(9) Distal solenophore branch $\mathbf{j}$ clearly larger than branch $\mathbf{k}$, a conspicuous ridge (z) proximal to both present (Figs 125-127). Xinjiang ............................... S. kabaki sp.n.

\section{Sellanucheza typica $\mathbf{s p . n .}$}

Figs 128-137.

HOLOTYPE OT (ZMUM), China, Sichuan Prov., Maoxian County, SE of Nanxinzhen, $3155 \mathrm{~m}$ a.s.1., $31^{\circ} 34^{\prime} 04^{\prime \prime} \mathrm{N}, 103^{\circ} 47^{\prime} 41^{\prime \prime} \mathrm{E}$, a small clearing in a broadleaved forest with a Rhododendron understorey, 30.06.2012, leg. I.A. Belousov \& G.E. Davidian.

NAME. To emphasize the typical facies of the new species failing to amend the concept of the genus.

DIAGNOSIS. Differs from all currently known species of the genus by the broadly truncate epiproct (Fig. 131), coupled with the solenophore being long, but broad, rather strongly coiled and deeply bifid, with both parabasal processes subunciform (see also Key and Remarks below).

DESCRIPTION. Length ca $39 \mathrm{~mm}$, width of midbody pro- and metazona 3.4 and $3.6 \mathrm{~mm}$. General coloration in alcohol rather uniformly light grey-brown to beige (Figs 128-131), but head and a few anteriormost segments a little more intensely so, partly even marbled; antennomeres 6 and 7 contrasting dark brown (Fig. 129), remaining antennomeres, legs and venter especially light, yellowish.

In width, head $=$ segments 3 and $4<$ collum $=$ segment $2<$ segments 5-16; thereafter, body gradually tapering towards telson. Clypeolabral region densely setose, vertigial region bare (Fig. 129). Antennae slender and rather long, extending behind metatergum 3 $\left(\mathrm{O}^{7}\right)$ when stretched dorsally; antennomeres 2-6 subequal in length. Paraterga very poorly developed (Figs 128-131 \& 133), set low, mostly lying at about half of midbody height; collum broadly and regularly rounded laterally; paraterga 2 broadly rounded and clearly extended frontally, more narrowly rounded and obtusangular (ca $120^{\circ}$ ) caudally, not projecting behind, declined more ventrad than laterad; following paraterga delimited by a faint and abbreviated sulcus only dorsally, in pore-bearing segments more evident due to ozopores borne on small tubercles (Figs 128 \& 133 ), but nearly fully obliterate in segments $17-19$. Ozopores lateral, placed at about caudal quarter of metatergite's length. Body surface smooth and shining. Axial line at most very vague. Transverse metatergal sulcus a thin line, slightly sinuate forward middorsally, barely visible in segment 4 , far from reaching bases of paraterga even in segments 5-18 (Figs $130,131 \& 133)$, absent from $19^{\text {th }}$. Tergal setae fully abraded, setation pattern untraceable. Stricture dividing pro- and metazona densely and finely striolate down to below paraterga (Fig. 133). Pleurosternal 


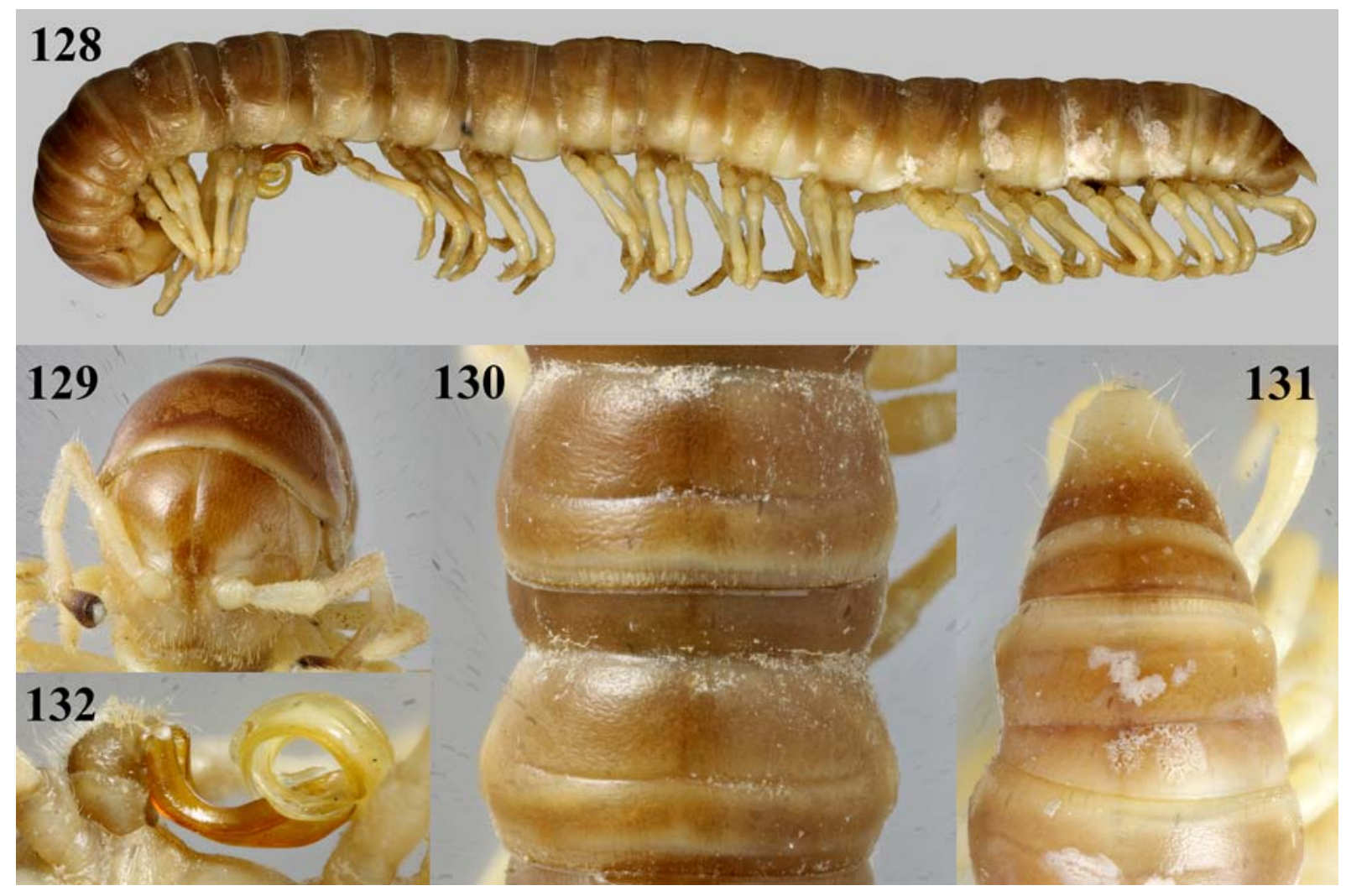

Figs 128-132. Sellanucheza typica sp.n., holotype: 128 - habitus, lateral; 129 - anterior body part, front; 130 - midbody segments, dorsal; 131 - caudal body part, dorsal; 132 - left gonopod in situ, lateral. Photos taken by K. Makarov, not to scale.

Рис. 128-132. Sellanucheza typica sp.n., голотип: 128 - общий вид, сбоку; 129 - передняя часть тела, спереди; $130-$ среднетуловищные сегменты, сверху; 131 - задняя часть тела, сверху; 132 - левый гонопод in situ, сбоку. Фото сделаны К. Макаровым, без масштаба.

carinae missing. Epiproct (Fig. 131) broadly truncate at tip, subapical lateral papillae evident. Hypoproct (Fig. 134) subtrapeziform, caudal 1+1 setae strongly separated, borne on highly inconspicuous knobs.

Sternites without modifications, nearly bare, near each coxa with a minute knob; cross-impressions very shallow; a densely setose, roundly subtrapeziform lamina between coxae 4 (Fig. 135). Legs (Figs 128 \& 133) slender, probably slightly incrassate and elongate in $\sigma^{7}$ compared to + , ca $1.7-1.8$ times $\left(O^{7}\right)$ as long as midbody height; $\sigma^{7}$ prefemora without evident lateral bulges; ventral brushes present in tibiae and tarsi until about legs of segment 12 , thereafter gradually thinning out; adenostyles absent.

Gonopods (Figs 132, 136 \& 137) rather complex; coxite relatively short, subcylindrical, slightly setose distoventrally; femorite long and slender, not twisted, modestly curved, supporting seminal groove only on its mesal side, about as long as acropodite, subapically with a moderately long spine (B) before a distinct sulcus demarcating a clearly coiled solenophore, the latter at base with a long mesal spine (A), apex rather deeply bifurcate into an outer spini- and an inner subspatuliform branch. A flagelliform solenomere about as long as solenophore.
REMARKS. Regrettably, in my previous contribution to the same series [Golovatch, 2013], I inadvertently missed the paper by Nguyen Duc [2011] which had treated the tribe Tonkinosomatini in Vietnam. The tribe has since been synonymized with Sulciferini, but my review of, as well as the key to, Sellanucheza species should have also contained S. hoffmani Nguyen Duc, 2011, another valid congener from northern Vietnam.

Sellanucheza Enghoff, Golovatch et Nguyen Duc, 2004 still remains a rather small genus of the tribe Sulciferini known to contain only six species: S. tenebra (Hoffman, 1961) (the type-species) and S. typica sp.n., both from Sichuan, China, S. jaegeri Golovatch, 2013, from Shaanxi, China, S. variata (Attems, 1953), S. grandis (Golovatch, 1984) and S. hoffmani Nguyen Duc, 2011, all three latter species from northern Vietnam [Nguyen Duc, 2011; Golovatch, 2013]. The new species fails to alter the generic diagnosis as formulated by Hoffman [1961, 1963], perhaps being especially similar to $S$. variata [see Hoffman, 1963; Golovatch, 1984] because of the particularly large size, as well as the coiled and subequally long gonopod femorite and solenophore (see Key below).

The following updated key can be proposed to separate all six known species of Sellanucheza. 


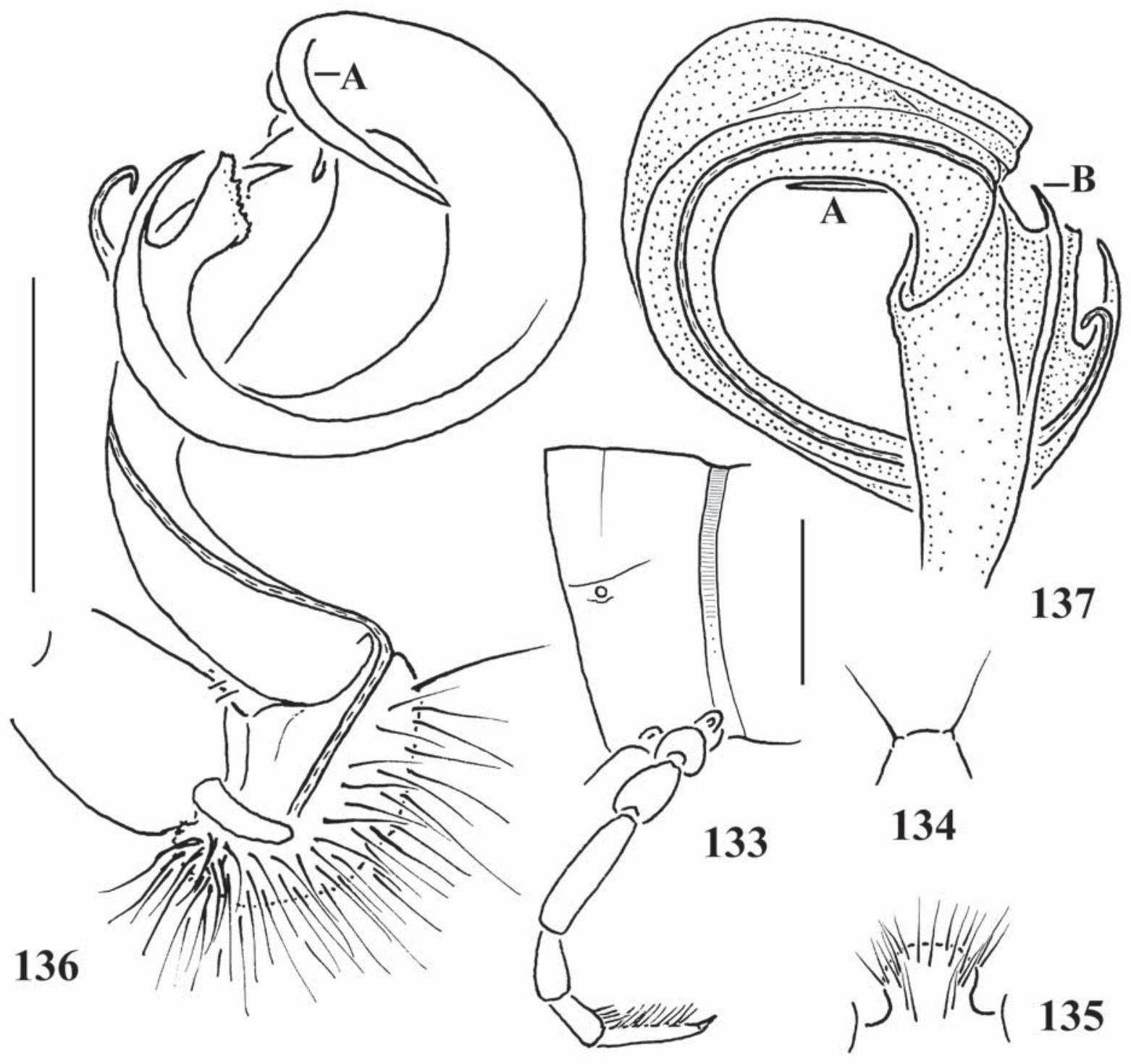

Figs 133-137. Sellanucheza typica sp.n., holotype: 133 - body segment 10, lateral; 134 - hypoproct, ventral; 135 - sternal lobe between coxae 4, caudoventral view; $136 \& 137$ - left gonopod, mesal and lateral views, respectively. Scale bars: $2.0(133-135)$ and 0.5 $\mathrm{mm}(136 \& 116)$.

Рис. 133-137. Sellanucheza typica sp.n., голотип: 133 - сегмент 10, сбоку; 134 - гипопрокт, снизу; 135 - стернальная пластина между тазиками 4, одновременно снизу и сзади; 136 и 137 - левый гонопод, соответственно изнутри и сбоку. Масштаб $2,0(133-135)$ и 0,5 мм (136 и 137$)$.

1(2) Paraterga 3-19 totally suppressed, at most faint bulges not delimited by a sulcus even dorsally. Solenophore long, about as long as a subunciform gonofemorite, strongly coiled, with two rather large, rounded, subequal lobes at apex. Sichuan, China ..................... S. tenebra

2(1) Paraterga, albeit poorly developed, mostly traceable as bulges delimited by a sulcus at least dorsally. Gonopod femorite more often, but not always, much slenderer and longer, being not so strongly curved. Solenophore usually shorter, often at least one apical branch spiniform ....... 3

3(4) Solenophore about as long as gonofemorite, clearly coiled, deeply bifid

4(3) Solenophore much, at least ca 2 times shorter than coiled gonofemorite
5(6) Gonofemorite elongate, slender, only slightly coiled (Figs 132,136 \& 137). Sichuan, China S. typica sp.n.

6(5) Gonofemorite shorter and stouter, far more strongly coiled. Laocai, Vietnam ..... S. variata

7(8) Paraterga, however small, complete, keel-shaped and delimited by distinct sulci both dorsally and ventrally. Spiniform process A of solenophore nearly straight .....

S. hoffmani

8(7) Paraterga abbreviated, delimited by a distinct sulcus only dorsally. Spiniform process A of solenophore clearly curved

9(10) Solenophore tip only faintly bifid, apical lobes small and rounded; solenophore near base with three outgrowths, process $\mathbf{B}$ being a small denticle, placed basal 
to both a lobe and process A. Ninhbinh, Vietnam ........ S. grandis

10(9) Solenophore tip deeply bifid, one apical branch spiniform; solenophore near base with two outgrowths, both processes $\mathbf{A}$ and $\mathbf{B}$ being long and subunciform. Shaanxi, China . S. jaegeri

ACKNOWLEDGEMENTS. I am extremely grateful to Igor Belousov, Ilya Kabak and Genrikh Davidian, all St. Petersburg, Russia, for allowing me to study most of the material treated above, as well as to deposit most of it in the ZMUM collection. Pavel Stoev (NMNHS) kindly provided on loan a fresh sample of Antheromorpha rosea for examination. The colour pictures were skillfully taken by K. Makarov, Moscow, Russia. The present project was supported in part through a grant released by the Russian Ministry of Education and Science.

\section{References}

Golovatch S.I. 1984. Contributions to the millipede fauna of Vietnam (Diplopoda) II. Acta Zool. Hung. Vol.30. Fasc.1-2. P.5377.

Golovatch S.I. 2009. On several new or poorly-known Oriental Paradoxosomatidae (Diplopoda: Polydesmida), VIII // Arthropoda Selecta. Vol.18. Nos 1/2. P.1-7.

Golovatch S.I. 2011. On several new or poorly-known Oriental Paradoxosomatidae (Diplopoda: Polydesmida), XI // Arthropoda Selecta. Vol.20. No.4. P.259-266.

Golovatch S.I. 2012. On several new or poorly-known Oriental Paradoxosomatidae (Diplopoda: Polydesmida), XII // Arthropoda Selecta. Vol.21. No.1. P.1-12.
Golovatch S.I. 2013. On several new or poorly-known Oriental Paradoxosomatidae (Diplopoda: Polydesmida), XIII // Arthropoda Selecta. Vol.22. No.1. P.1-31.

Hoffman R.L. 1961. Two new diplopod genera from western China (Polydesmida: Strongylosomatidae) // Annals and Magazine of Natural History, Ser.13. Vol.3. P.533-543.

Hoffman R.L. 1963. A contribution to the knowledge of Asiatic strongylosomoid Diplopoda (Polydesmida: Strongylosomatidae) // Annals and Magazine of Natural History, Ser.13. Vol.5. P.577-593.

Hoffman R.L. 1980. Classification of the Diplopoda // Muséum d'histoire naturelle, Genève. 237 pp. [for 1979].

Jeekel C.A.W. 1968. On the classification and geographical distribution of the family Paradoxosomatidae (Diplopoda, Polydesmida) // Privately published. Academisch Proefschrift (PhD Thesis), Rotterdam. 162 pp.

Jeekel C.A.W. 1988. The generic position of Orthomorpha bucharensis Lohmander and $O$. muminabadensis Gulička, and the taxonomic status of Hedinomorpha Verhoeff (Diplopoda, Polydesmida, Paradoxosomatidae) // Bulletin Zoölogisch Museum Universiteit van Amsterdam. Vol.11. No.11. P.97-104.

Nguyen Duc A. 2011. A review of the millipede tribe Tonkinosomatini (Diplopoda: Polydesmida: Paradoxosomatidae) from Vietnam // Zootaxa. No.3036. P.58-68.

Verhoeff K.W. 1933. Schwedisch-chinesische wissenschaftliche Expedition nach den nordwestlichen Provinzen Chinas, unter Leitung von Dr. Sven Hedin und Prof. Sü Ping-Chang. Myriapoda gesammelt vom schwedischen Arzt der Expedition Dr. David Hummel 1927-1930 // Ark. Zool. Bd.26A. Nr.10. S.1-41.

Zhang C.Z., Tang H.Q. 1985. Eine neue Art der Gattung Hedinomorpha (Diplopoda, Paradoxosomatidae) aus China // Sinozoologia. No.3. P.35-38 [in Chinese, summary in German].

Responsible editor K.G. Mikhailov 